\title{
Numerical analysis of the Navier-Stokes/Darcy coupling
}

\author{
Lori Badea $^{1}$, Marco Discacciati ${ }^{2}$, Alfio Quarteroni ${ }^{2,3}$
}

1. Institute of Mathematics of the Romanian Academy,

P.O. Box 1-764, 014700 Bucharest, Romania. Lori.Badea@imar.ro

2. IACS - Chair of Modeling and Scientific Computing, EPFL, CH-1015 Lausanne,

Switzerland. marco.discacciati@epfl.ch, alfio.quarteroni@epfl.ch

3. MOX, Politecnico di Milano, P.zza Leonardo da Vinci 32, I-20133 Milano, Italy.

\begin{abstract}
We consider a differential system based on the coupling of the Navier-Stokes and Darcy equations for modeling the interaction between surface and porousmedia flows. We formulate the problem as an interface equation, we analyze the associated (nonlinear) Steklov-Poincaré operators, and we prove its wellposedness. We propose and analyze iterative methods to solve a conforming finite element approximation of the coupled problem.
\end{abstract}

\section{Introduction and problem setting}

Let $\Omega \subset \mathbb{R}^{d}(d=2,3)$ be a bounded domain, decomposed into two non intersecting subdomains $\Omega_{f}$ and $\Omega_{p}$ separated by an interface $\Gamma$, i.e. $\bar{\Omega}=\bar{\Omega}_{f} \cup \bar{\Omega}_{p}$, $\Omega_{f} \cap \Omega_{p}=\emptyset$ and $\bar{\Omega}_{f} \cap \bar{\Omega}_{p}=\Gamma$. We suppose the boundaries $\partial \Omega_{f}$ and $\partial \Omega_{p}$ to be Lipschitz continuous. From the physical point of view, $\Gamma$ is a surface separating the domain $\Omega_{f}$ filled by a fluid, from a domain $\Omega_{p}$ formed by a porous medium. We assume that the fluid contained in $\Omega_{f}$ has a fixed surface (i.e. we do not consider the free surface fluid case) and can filtrate through the adjacent porous medium. See for example Fig. 1.

In this paper, we will refer explicitely to the hydraulic situation of Fig. 1 left. However, the mathematical results that we present can apply with minor changes also to the more general framework introduced above (see Remark 1.1). In order to describe the motion of the fluid in $\Omega_{f}$, we introduce the Navier-Stokes equations: $\forall t>0$,

$$
\begin{aligned}
& \partial_{t} \mathbf{u}_{f}-\boldsymbol{\nabla} \cdot \mathbf{T}\left(\mathbf{u}_{f}, p_{f}\right)+\left(\mathbf{u}_{f} \cdot \nabla\right) \mathbf{u}_{f}=\mathbf{f} \quad \text { in } \Omega_{f}, \\
& \nabla \cdot \mathbf{u}_{f}=0 \quad \text { in } \Omega_{f} \text {, }
\end{aligned}
$$

where $\mathbf{T}\left(\mathbf{u}_{f}, p_{f}\right)=\nu\left(\nabla \mathbf{u}_{f}+\nabla^{T} \mathbf{u}_{f}\right)-p_{f} \mathbf{l}$ is the Cauchy stress tensor, $\nu>0$ is the kinematic viscosity of the fluid, while $\mathbf{u}_{f}$ and $p_{f}$ are the fluid velocity and pressure, respectively; $\nabla$ is the gradient operator with respect to the space coordinates. 

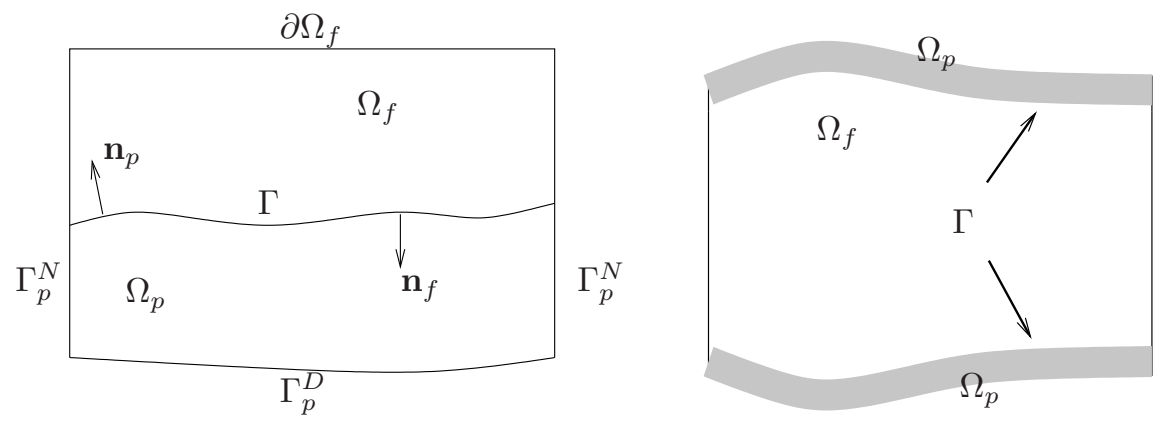

Figure 1: Schematic representation of a 2D section of possible computational domains: the surface-groundwater setting on the left, and the blood-flow problem on the right.

In the domain $\Omega_{p}$ we define the piezometric head $\varphi=z+p_{p} /\left(\rho_{f} g\right)$, where $z$ is the elevation from a reference level, $p_{p}$ is the pressure of the fluid in $\Omega_{p}, \rho_{f}$ its density and $g$ is the gravity acceleration.

The fluid motion in $\Omega_{p}$ is described by the equations:

$$
\begin{aligned}
n \mathbf{u}_{p} & =-K \nabla \varphi & & \text { in } \Omega_{p} \\
\nabla \cdot \mathbf{u}_{p} & =0 & & \text { in } \Omega_{p}
\end{aligned}
$$

where $\mathbf{u}_{p}$ is the fluid velocity, $n$ is the volumetric porosity and $K$ is the hydraulic conductivity tensor $K=\operatorname{diag}\left(K_{1}, \ldots, K_{d}\right)$ with $K_{i} \in L^{\infty}\left(\Omega_{p}\right), i=1, \ldots, d$. The first equation is Darcy's law. In the following we shall denote $\mathrm{K}=K / n=$ $\operatorname{diag}\left(K_{i} / n\right)(i=1, \ldots, d)$. Darcy's law provides the simplest linear relation between velocity and pressure in porous media under the physically reasonable assumption that fluid flows are usually very slow and all the inertial (nonlinear) terms may be neglected. Extensions of Darcy's law are given, e.g., by the Forchheimer's or Brinkman's equations when the Reynolds number in $\Omega_{p}$ is not small (see $[15,16,24,8]$ ), or by more complicated models like Richards' equations apt to describe saturated-unsaturated fluid flows (see, e.g., [3] and references therein).

For the sake of clarity, in our analysis we shall adopt homogeneous boundary conditions. The treatment of non-homogeneous conditions involves some additional technicalities, but neither the guidelines of the theory nor the final results are affected. We refer to [10]. In particular, for the Navier-Stokes problem we impose the no-slip condition $\mathbf{u}_{f}=\mathbf{0}$ on $\partial \Omega_{f} \backslash \Gamma$, while for the Darcy problem, we set the piezometric head $\varphi=0$ on $\Gamma_{p}^{D}$ and we require the normal velocity to be null on $\Gamma_{p}^{N}, \mathbf{u}_{p} \cdot \mathbf{n}_{p}=0$ on $\Gamma_{p}^{N}$, where $\partial \Omega_{p}=\Gamma \cup \Gamma_{p}^{D} \cup \Gamma_{p}^{N}$ (see Fig. 1, left). $\mathbf{n}_{p}$ and $\mathbf{n}_{f}$ denote the unit outward normal vectors to the surfaces $\partial \Omega_{p}$ and $\partial \Omega_{f}$, respectively, and we have $\mathbf{n}_{f}=-\mathbf{n}_{p}$ on $\Gamma$. We suppose $\mathbf{n}_{f}$ and $\mathbf{n}_{p}$ to be regular enough. In the following we shall indicate $\mathbf{n}=\mathbf{n}_{f}$ for simplicity of notation.

We supplement the Navier-Stokes and Darcy problems with the following con- 
ditions on $\Gamma$ :

$$
\begin{aligned}
\mathbf{u}_{p} \cdot \mathbf{n} & =\mathbf{u}_{f} \cdot \mathbf{n}, \\
-\mathbf{n} \cdot\left(\mathbf{T}\left(\mathbf{u}_{f}, p_{f}\right) \cdot \mathbf{n}\right) & =g \varphi, \\
-\varepsilon \boldsymbol{\tau}_{i} \cdot\left(\mathbf{T}\left(\mathbf{u}_{f}, p_{f}\right) \cdot \mathbf{n}\right) & =\nu \mathbf{u}_{f} \cdot \boldsymbol{\tau}_{i}, \quad i=1, \ldots, d-1,
\end{aligned}
$$

where $\boldsymbol{\tau}_{i}(i=1, \ldots, d-1)$ are linear independent unit tangential vectors to the boundary $\Gamma$, and $\varepsilon$ is the characteristic length of the pores of the porous medium.

Conditions (3) and (4) impose the continuity of the normal velocity on $\Gamma$, as well as that of the normal component of the normal stress, however they allow pressure to be discontinuous across the interface. The so-called Beavers-JosephSaffman condition (5) does not yield any coupling. Indeed, it provides a boundary condition for the Navier-Stokes problem since it involves only quantities in the domain $\Omega_{f}$.

A mathematical justification of these interface conditions can be found in [18, 19, 20]. The same interface conditions have been considered in [11, 12, 13, 22] for the coupling of Stokes and Darcy equations.

Remark 1.1 Our results could be extended, e.g., to the filtration of blood through the arterial wall. Indeed, the wall of the blood vessel can be described as a porous media replacing the piezometric head in (2) by the blood pressure. The coupling conditions (3)-(4) would apply as well since they require the continuity of normal fluxes and that of normal stresses, as investigated in [28]. Only condition (5) could be simplified requiring $\mathbf{u}_{f} \cdot \boldsymbol{\tau}_{i}=0$ on $\Gamma$, but this would not significantly modify the coupling as this condition is simply a boundary condition for the fluid problem in $\Omega_{f}$.

From now on, we focus on the steady problem obtained by dropping the time derivative in the momentum equation (1). This can be motivated by, e.g., the use of an implicit time-advancing scheme on the time-dependent problem (1). Moreover, instead of (2), we consider the following equivalent formulation for Darcy problem:

$$
\text { find } \varphi: \quad-\nabla \cdot(K \nabla \varphi)=0 \quad \text { in } \Omega_{p} .
$$

We define the following functional spaces:

$$
\begin{aligned}
& H_{f}=\left\{\mathbf{v} \in\left(H^{1}\left(\Omega_{f}\right)\right)^{d}: \mathbf{v}=\mathbf{0} \text { on } \partial \Omega_{f} \backslash \Gamma\right\}, \\
& H_{f}^{0}=\left\{\mathbf{v} \in H_{f}: \mathbf{v} \cdot \mathbf{n}=0 \text { on } \Gamma\right\}, \\
& V_{f}=\left\{\mathbf{v} \in H_{f}: \nabla \cdot \mathbf{v}=0 \text { in } \Omega_{f}\right\}, V_{f}^{0}=\left\{\mathbf{v} \in H_{f}^{0}: \nabla \cdot \mathbf{v}=0 \text { in } \Omega_{f}\right\}, \\
& H_{p}=\left\{\psi \in H^{1}\left(\Omega_{p}\right): \psi=0 \text { on } \Gamma_{p}^{D}\right\}, H_{p}^{0}=\left\{\psi \in H_{p}: \psi=0 \text { on } \Gamma\right\}, \\
& Q=L^{2}\left(\Omega_{f}\right), Q_{0}=\left\{q \in Q: \int_{\Omega_{f}} q=0\right\} .
\end{aligned}
$$

We denote by $|\cdot|_{1}$ and $\|\cdot\|_{1}$ the $H^{1}$-seminorm and norm, respectively, and by $\|\cdot\|_{0}$ the $L^{2}$-norm; it will always be clear form the context whether we are referring to spaces on $\Omega_{f}$ or $\Omega_{p}$.

Finally, we consider the trace space $\Lambda=H_{00}^{1 / 2}(\Gamma)$ (see [23]) and its subspace $\Lambda_{0}=\left\{\mu \in \Lambda: \int_{\Gamma} \mu=0\right\}$. 
Then, we introduce the bilinear forms

$$
\begin{array}{ll}
a_{f}(\mathbf{v}, \mathbf{w})=\int_{\Omega_{f}} \frac{\nu}{2}\left(\nabla \mathbf{v}+\nabla^{T} \mathbf{v}\right) \cdot\left(\nabla \mathbf{w}+\nabla^{T} \mathbf{w}\right) \quad \forall \mathbf{v}, \mathbf{w} \in\left(H^{1}\left(\Omega_{f}\right)\right)^{d}, \\
b_{f}(\mathbf{v}, q)=-\int_{\Omega_{f}} q \nabla \cdot \mathbf{v} \quad \forall \mathbf{v} \in\left(H^{1}\left(\Omega_{f}\right)\right)^{d}, \quad \forall q \in Q, \\
a_{p}(\varphi, \psi)=\int_{\Omega_{p}} \nabla \psi \cdot \mathrm{K} \nabla \varphi \quad \forall \varphi, \psi \in H^{1}\left(\Omega_{p}\right),
\end{array}
$$

and, for all $\mathbf{v}, \mathbf{w}, \mathbf{z} \in\left(H^{1}\left(\Omega_{f}\right)\right)^{d}$, the trilinear form

$$
c_{f}(\mathbf{w} ; \mathbf{z}, \mathbf{v})=\int_{\Omega_{f}}[(\mathbf{w} \cdot \nabla) \mathbf{z}] \cdot \mathbf{v}=\sum_{i, j=1}^{d} \int_{\Omega_{f}} w_{j} \frac{\partial z_{i}}{\partial x_{j}} v_{i} .
$$

The coupling conditions (3)-(4) can be incorporated in the weak form of the Navier-Stokes/Darcy problem as natural conditions on $\Gamma$. In fact, the weak formulation reads:

find $\mathbf{u}_{f} \in H_{f}, p_{f} \in Q, \varphi \in H_{p}$ such that

$$
\begin{aligned}
& a_{f}\left(\mathbf{u}_{f}, \mathbf{v}\right)+c_{f}\left(\mathbf{u}_{f} ; \mathbf{u}_{f}, \mathbf{v}\right)+b_{f}\left(\mathbf{v}, p_{f}\right) \\
& +\int_{\Gamma} g \varphi(\mathbf{v} \cdot \mathbf{n})+\int_{\Gamma} \sum_{j=1}^{d-1} \frac{\nu}{\varepsilon}\left(\mathbf{u}_{f} \cdot \boldsymbol{\tau}_{j}\right)\left(\mathbf{v} \cdot \boldsymbol{\tau}_{j}\right)=\int_{\Omega_{f}} \mathbf{f} \cdot \mathbf{v}, \\
& b_{f}\left(\mathbf{u}_{f}, q\right)=0, \\
& a_{p}(\varphi, \psi)=\int_{\Gamma} \psi\left(\mathbf{u}_{f} \cdot \mathbf{n}\right),
\end{aligned}
$$

for all $\mathbf{v} \in H_{f}, q \in Q, \psi \in H_{p}$.

The rest of the paper is organized as follows. In Sect. 2, we introduce and analyze some nonlinear extension operator that will be used in Sect. 3 to reformulate the coupled problem (16)-(18) as a nonlinear interface equation, say $\mathcal{S}(\lambda)=$ 0 , whose sole scalar unknown $\lambda$ is the common value of the normal velocity $\mathbf{u}_{f} \cdot \mathbf{n}=\mathbf{u}_{p} \cdot \mathbf{n}$ across $\Gamma . \mathcal{S}$ is a nonlinear Steklov-Poincaré operator. The well-posedness of this interface problem is proved in Sect. 3. Finally, in Sect. 4, after briefly discussing a conforming finite element approximation of the NavierStokes/Darcy problem, we propose and analyze three different iterative methods to compute its solution.

\section{Some nonlinear extension operators: defini- tion and analysis}

In this section we apply domain decomposition techniques at the differential level to study the Navier-Stokes/Darcy problem, as done in an abstract setting in [25]. We identify the subdomains with $\Omega_{f}$ and $\Omega_{p}$, then we introduce and analyze some nonlinear extension operators that will be used in Sect. 3 to write the Steklov-Poincaré interface equation associated to the coupled problem. 
We consider two linear continuous extension operators:

$$
\begin{aligned}
& \boldsymbol{R}^{1}: \Lambda \rightarrow H_{f} \quad \text { s.t. } \quad \boldsymbol{R}^{1} \mu \cdot \mathbf{n}=\mu \text { on } \Gamma, \quad \forall \mu \in \Lambda, \\
& R^{2}: H^{1 / 2}(\Gamma) \rightarrow H_{p} \quad \text { s.t. } \quad R^{2} \mu=\mu \text { on } \Gamma, \quad \forall \mu \in H^{1 / 2}(\Gamma) .
\end{aligned}
$$

Since there holds $H_{f}=H_{f}^{0}+\left\{\boldsymbol{R}_{f}^{1} \mu: \mu \in \Lambda\right\}$, we can prove the following result (see also [10]).

Proposition 2.1 The coupled Navier-Stokes/Darcy problem (16)-(18) can be equivalently reformulated in the multidomain form:

find $\mathbf{u}_{f} \in H_{f}, p_{f} \in Q, \varphi \in H_{p}$ such that

$$
\begin{aligned}
& a_{f}\left(\mathbf{u}_{f}, \mathbf{v}\right)+c_{f}\left(\mathbf{u}_{f} ; \mathbf{u}_{f}, \mathbf{v}\right)+b_{f}\left(\mathbf{v}, p_{f}\right) \\
& \quad+\int_{\Gamma} \sum_{j=1}^{d-1} \frac{\nu}{\varepsilon}\left(\mathbf{u}_{f} \cdot \boldsymbol{\tau}_{j}\right)\left(\mathbf{v} \cdot \boldsymbol{\tau}_{j}\right)=\int_{\Omega_{f}} \mathbf{f} \cdot \mathbf{v} \quad \forall \mathbf{v} \in H_{f}^{0}, \\
& b_{f}\left(\mathbf{u}_{f}, q\right)=0 \quad \forall q \in Q, \\
& a_{p}(\varphi, \psi)=0 \quad \forall \psi \in H_{p}^{0}, \\
& \int_{\Gamma}\left(\mathbf{u}_{f} \cdot \mathbf{n}\right) \mu=a_{p}\left(\varphi, R^{2} \mu\right) \quad \forall \mu \in \Lambda, \\
& \int_{\Gamma} g \varphi \mu=\int_{\Omega_{f}} \mathbf{f} \cdot\left(\boldsymbol{R}_{f}^{1} \mu\right)-a_{f}\left(\mathbf{u}_{f}, \boldsymbol{R}^{1} \mu\right)-c_{f}\left(\mathbf{u}_{f} ; \mathbf{u}_{f}, \boldsymbol{R}^{1} \mu\right) \\
& \quad-b_{f}\left(\boldsymbol{R}^{1} \mu, p_{f}\right)-\int_{\Gamma} \sum_{j=1}^{d-1} \frac{\nu}{\varepsilon}\left(\mathbf{u}_{f} \cdot \boldsymbol{\tau}_{j}\right)\left(\boldsymbol{R}^{1} \mu \cdot \boldsymbol{\tau}_{j}\right) \quad \forall \mu \in \Lambda .
\end{aligned}
$$

We would like to rewrite (21)-(25) as an interface equation in a scalar interface unknown defined on $\Gamma$ corresponding to the trace of the fluid normal velocity $\mathbf{u}_{f} \cdot \mathbf{n}$ on $\Gamma$. First of all, we need to introduce and analyze some further extension operators.

Let us consider the (unknown) interface variable $\lambda=\left(\mathbf{u}_{f} \cdot \mathbf{n}\right)_{\mid \Gamma}$. Due to the incompressibility constraint in $\Omega_{f}$ and to the boundary conditions imposed on $\partial \Omega_{f} \backslash \Gamma$, it must be $\lambda \in \Lambda_{0}$.

Let us define the linear extension operator:

$$
R_{f}: \Lambda_{0} \rightarrow H_{f} \times Q_{0}, \quad \eta \rightarrow R_{f} \eta=\left(\boldsymbol{R}_{f}^{1} \eta, R_{f}^{2} \eta\right)
$$

satisfying $\boldsymbol{R}_{f}^{1} \eta \cdot \mathbf{n}=\eta$ on $\Gamma$, and, for all $\mathbf{v} \in H_{f}^{0}, q \in Q_{0}$,

$$
\begin{array}{r}
a_{f}\left(\boldsymbol{R}_{f}^{1} \eta, \mathbf{v}\right)+b_{f}\left(\mathbf{v}, R_{f}^{2} \eta\right)+\int_{\Gamma} \sum_{j=1}^{d-1} \frac{\nu}{\varepsilon}\left(\boldsymbol{R}_{f}^{1} \eta \cdot \boldsymbol{\tau}_{j}\right)\left(\mathbf{v} \cdot \boldsymbol{\tau}_{j}\right)=0 \\
b_{f}\left(\boldsymbol{R}_{f}^{1} \eta, q\right)=0 .
\end{array}
$$

Moreover, we consider the linear extension operator

$$
R_{p}: \Lambda_{0} \rightarrow H_{p}, \quad \eta \rightarrow R_{p} \eta
$$

such that

$$
a_{p}\left(R_{p} \eta, \psi\right)=\int_{\Gamma} \eta \psi \quad \forall \psi \in H_{p}
$$


It is easy to see that problems (27)-(28) and (30) both have a unique solution. Finally, let us introduce the following nonlinear extension operator:

$$
\mathcal{R}_{f}: \Lambda_{0} \rightarrow H_{f} \times Q_{0}, \quad \eta \rightarrow \mathcal{R}_{f}(\eta)=\left(\mathcal{R}_{f}^{1}(\eta), \mathcal{R}_{f}^{2}(\eta)\right)
$$

such that $\boldsymbol{\mathcal { R }}_{f}^{1}(\eta) \cdot \mathbf{n}=\eta$ on $\Gamma$, and, for all $\mathbf{v} \in H_{f}^{0}, q \in Q_{0}$,

$$
\begin{aligned}
a_{f}\left(\boldsymbol{\mathcal { R }}_{f}^{1}(\eta), \mathbf{v}\right)+c_{f}\left(\boldsymbol{\mathcal { R }}_{f}^{1}(\eta) ; \boldsymbol{\mathcal { R }}_{f}^{1}(\eta), \mathbf{v}\right)+b_{f}\left(\mathbf{v}, \mathcal{R}_{f}^{2}(\eta)\right) & \\
+\int_{\Gamma} \sum_{j=1}^{d-1} \frac{\nu}{\varepsilon}\left(\boldsymbol{\mathcal { R }}_{f}^{1}(\eta) \cdot \boldsymbol{\tau}_{j}\right)\left(\mathbf{v} \cdot \boldsymbol{\tau}_{j}\right) & =\int_{\Omega_{f}} \mathbf{f} \cdot \mathbf{v}, \\
b_{f}\left(\boldsymbol{\mathcal { R }}_{f}^{1}(\eta), q\right) & =0 .
\end{aligned}
$$

In order to prove the existence and uniqueness of $\mathcal{R}_{f}$, we define the auxiliary nonlinear operator

$$
\begin{aligned}
& \mathcal{R}_{0}: \Lambda_{0} \rightarrow H_{f}^{0} \times Q_{0}, \quad \eta \rightarrow \mathcal{R}_{0}(\eta)=\left(\mathcal{R}_{0}^{1}(\eta), \mathcal{R}_{0}^{2}(\eta)\right), \\
& \text { with } \mathcal{R}_{0}^{i}(\eta)=\mathcal{R}_{f}^{i}(\eta)-R_{f}^{i} \eta, \quad i=1,2 .
\end{aligned}
$$

Clearly, $\mathcal{R}_{0}^{1}(\eta) \cdot \mathbf{n}=0$ on $\Gamma$, and it satisfies:

$$
\begin{aligned}
a_{f}\left(\boldsymbol{\mathcal { R }}_{0}^{1}(\eta), \mathbf{v}\right)+c_{f}\left(\boldsymbol{R}_{f}^{1} \eta+\mathcal{R}_{0}^{1}(\eta) ; \boldsymbol{R}_{f}^{1} \eta+\mathcal{R}_{0}^{1}(\eta), \mathbf{v}\right) & \\
+b_{f}\left(\mathbf{v}, \mathcal{R}_{0}^{2}(\eta)\right)+\int_{\Gamma} \sum_{j=1}^{d-1} \frac{\nu}{\varepsilon}\left(\boldsymbol{\mathcal { R }}_{0}^{1}(\eta) \cdot \boldsymbol{\tau}_{j}\right)\left(\mathbf{v} \cdot \boldsymbol{\tau}_{j}\right) & =\int_{\Omega_{f}} \mathbf{f} \cdot \mathbf{v} \\
b_{f}\left(\boldsymbol{\mathcal { R }}_{0}^{1}(\eta), q\right) & =0
\end{aligned}
$$

for all $\mathbf{v} \in H_{f}^{0}, q \in Q_{0}$. Remark that problem (34)-(35) is analogous to (31)(32), but here $\mathcal{R}_{0}^{1}(\eta) \in H_{f}^{0}$, while $\mathcal{R}_{f}^{1}(\eta) \in H_{f}$.

Moreover, given $\eta \in \Lambda_{0}$, we define the form

$$
\begin{aligned}
& a(\mathbf{w} ; \mathbf{z}, \mathbf{v})=a_{f}(\mathbf{z}, \mathbf{v})+c_{f}(\mathbf{w} ; \mathbf{z}, \mathbf{v})+c_{f}\left(\boldsymbol{R}_{f}^{1} \eta ; \mathbf{z}, \mathbf{v}\right) \\
& \quad+c_{f}\left(\mathbf{z} ; \boldsymbol{R}_{f}^{1} \eta, \mathbf{v}\right)+\int_{\Gamma} \sum_{j=1}^{d-1} \frac{\nu}{\varepsilon}\left(\mathbf{z} \cdot \boldsymbol{\tau}_{j}\right)\left(\mathbf{v} \cdot \boldsymbol{\tau}_{j}\right) \quad \forall \mathbf{w}, \mathbf{z}, \mathbf{v} \in\left(H^{1}\left(\Omega_{f}\right)\right)^{d},
\end{aligned}
$$

and the functional

$$
\langle\ell, \mathbf{v}\rangle=-c_{f}\left(\boldsymbol{R}_{f}^{1} \eta ; \boldsymbol{R}_{f}^{1} \eta, \mathbf{v}\right)+\int_{\Omega_{f}} \mathbf{f} \cdot \mathbf{v} \quad \forall \mathbf{v} \in\left(H^{1}\left(\Omega_{f}\right)\right)^{d} .
$$

Thus, we can rewrite (34)-(35) as: given $\eta \in \Lambda_{0}$,

$$
\text { find } \mathcal{R}_{0}^{1}(\eta) \in V_{f}^{0}: \quad a\left(\mathcal{R}_{0}^{1}(\eta) ; \mathcal{R}_{0}^{1}(\eta), \mathbf{v}\right)=\langle\ell, \mathbf{v}\rangle \quad \forall \mathbf{v} \in V_{f}^{0} .
$$

Finally, let us recall some useful inequalities: the Poincaré inequality (see, e.g., [25], p. 11)

$$
\exists C_{\Omega_{f}}>0: \quad\|\mathbf{v}\|_{0} \leq C_{\Omega_{f}}|\mathbf{v}|_{1} \quad \forall \mathbf{v} \in H_{f},
$$


the Korn inequality (see, e.g., [26], p. 149)

$$
\exists C_{\kappa}>0: \int_{\Omega_{f}} \sum_{i, j=1}^{d}\left(\frac{\partial v_{j}}{\partial x_{i}}+\frac{\partial v_{i}}{\partial x_{j}}\right)^{2} \geq C_{\kappa}\|\mathbf{v}\|_{1}^{2} \quad \forall \mathbf{v}=\left(v_{1}, \ldots, v_{d}\right) \in H_{f},
$$

and the following inequality

$$
\exists C_{\mathcal{N}}>0: \quad\left|c_{f}(\mathbf{w} ; \mathbf{z}, \mathbf{v})\right| \leq C_{\mathcal{N}}|\mathbf{w}|_{1}|\mathbf{z}|_{1}|\mathbf{v}|_{1} \quad \forall \mathbf{w}, \mathbf{z}, \mathbf{v} \in H_{f},
$$

which follows from the Poincaré inequality (39) and the inclusion $\left(H^{1}\left(\Omega_{f}\right)\right)^{d} \subset$ $\left(L^{4}\left(\Omega_{f}\right)\right)^{d}$ (for $d=2,3$ ) due to the Sobolev embedding theorem (see [1]).

We can now state the following result.

Proposition 2.2 Let $\mathbf{f} \in L^{2}\left(\Omega_{f}\right)$ be such that

$$
C_{\mathcal{N}} C_{\Omega_{f}}\|\mathbf{f}\|_{0}<\left(\frac{C_{\kappa} \nu}{2}\right)^{2}
$$

where $C_{\kappa}$ and $C_{\mathcal{N}}$ are the constants introduced in (40) and (41), respectively. If

$$
\eta \in\left\{\mu \in \Lambda_{0}:\left|\boldsymbol{R}_{f}^{1} \mu\right|_{1}<\frac{C_{\kappa} \nu-\sqrt{\left(\frac{C_{\kappa} \nu}{2}\right)^{2}+3 C_{\mathcal{N}} C_{\Omega}|| \mathbf{f} \|_{0}}}{3 C_{\mathcal{N}}}\right\},
$$

then there exists a unique nonlinear extension $\mathcal{R}_{f}(\eta)=\left(\mathcal{R}_{f}^{1}(\eta), \mathcal{R}_{f}^{2}(\eta)\right) \in H_{f} \times$ $Q_{0}$.

Remark 2.1 Notice that (43) imposes a constraint on $\eta$. In particular, since the norms $\left|\boldsymbol{R}_{f}^{1} \eta\right|_{1}$ and $\|\eta\|_{\Lambda}$ are equivalent (see [12], Lemma 4.1), this condition implies that a unique extension $\mathcal{R}_{f}(\eta)$ exists, provided the norm of $\eta$ is small enough. In our specific case, this means that we would be able to consider an extension $\mathcal{R}_{f}(\lambda)$ only if the normal velocity $\lambda$ across the interface $\Gamma$ is sufficiently small. Finally, remark that (42) guarantees that the radius of the ball in (43) is positive.

Proof. The proof is made of several steps and it is based on Theorems 6.1-6.2.

1. Let $\mathbf{v}, \mathbf{w} \in V_{f}^{0}$ and $\eta \in \Lambda_{0}$. Then, we have

$$
\begin{aligned}
a(\mathbf{w} ; \mathbf{v}, \mathbf{v})= & a_{f}(\mathbf{v}, \mathbf{v})+c_{f}(\mathbf{w} ; \mathbf{v}, \mathbf{v}) \\
& +c_{f}\left(\boldsymbol{R}_{f}^{1} \eta ; \mathbf{v}, \mathbf{v}\right)+c_{f}\left(\mathbf{v} ; \boldsymbol{R}_{f}^{1} \eta, \mathbf{v}\right)+\int_{\Gamma} \sum_{j=1}^{d-1} \frac{\nu}{\varepsilon}\left(\mathbf{v} \cdot \boldsymbol{\tau}_{j}\right)\left(\mathbf{v} \cdot \boldsymbol{\tau}_{j}\right) .
\end{aligned}
$$

Integrating by parts and recalling that $\mathbf{w} \in V_{f}^{0}$, then

$$
c_{f}(\mathbf{w} ; \mathbf{v}, \mathbf{v})=\frac{1}{2} \int_{\partial \Omega_{f}} \mathbf{w} \cdot \mathbf{n}|\mathbf{v}|^{2}-\frac{1}{2} \int_{\Omega_{f}} \nabla \cdot \mathbf{w}|\mathbf{v}|^{2}=0,
$$


where $|\mathbf{v}|$ is the Euclidean norm of the vector $\mathbf{v}$. Since

$$
\int_{\Gamma} \sum_{j=1}^{d-1} \frac{\nu}{\varepsilon}\left(\mathbf{v} \cdot \boldsymbol{\tau}_{j}\right)\left(\mathbf{v} \cdot \boldsymbol{\tau}_{j}\right) \geq 0
$$

from (44) we get

$$
a(\mathbf{w} ; \mathbf{v}, \mathbf{v}) \geq a_{f}(\mathbf{v}, \mathbf{v})+c_{f}\left(\boldsymbol{R}_{f}^{1} \eta ; \mathbf{v}, \mathbf{v}\right)+c_{f}\left(\mathbf{v} ; \boldsymbol{R}_{f}^{1} \eta, \mathbf{v}\right)
$$

and using the inequalities (40) and (41) we obtain

$$
a(\mathbf{w} ; \mathbf{v}, \mathbf{v}) \geq \frac{C_{\kappa} \nu}{2}|\mathbf{v}|_{1}^{2}-2 C_{\mathcal{N}}|\mathbf{v}|_{1}^{2}\left|\boldsymbol{R}_{f}^{1} \eta\right|_{1} .
$$

Then, thanks to (43), the bilinear form $a(\mathbf{w} ; \cdot, \cdot)$ is uniformly elliptic on $V_{f}^{0}$ with respect to $\mathbf{w}$, with constant $\alpha_{a}$ (independent of $\mathbf{w}$ )

$$
\alpha_{a}=\frac{C_{\kappa} \nu}{2}-2 C_{\mathcal{N}}\left|\boldsymbol{R}_{f}^{1} \eta\right|_{1} .
$$

2. Still using (41), we easily obtain:

$$
\left|a\left(\mathbf{w}_{1} ; \mathbf{z}, \mathbf{v}\right)-a\left(\mathbf{w}_{2} ; \mathbf{z}, \mathbf{v}\right)\right|=\left|c_{f}\left(\mathbf{w}_{1}-\mathbf{w}_{2} ; \mathbf{z}, \mathbf{v}\right)\right| \leq C_{\mathcal{N}}\left|\mathbf{w}_{1}-\mathbf{w}_{2}\right|_{1}|\mathbf{v}|_{1}|\mathbf{z}|_{1} .
$$

3. We have

$$
\begin{aligned}
\|\Pi \ell\|_{\left(V_{f}^{0}\right)^{\prime}} & =\sup _{\mathbf{v} \in V_{f}^{0}, \mathbf{v} \neq 0} \frac{\left|-c_{f}\left(\boldsymbol{R}_{f}^{1} \eta ; \boldsymbol{R}_{f}^{1} \eta, \mathbf{v}\right)+\int_{\Omega_{f}} \mathbf{f} \cdot \mathbf{v}\right|}{|\mathbf{v}|_{1}} \\
& \leq \sup _{\mathbf{v} \in V_{f}^{0}, \mathbf{v} \neq 0} \frac{C_{\mathcal{N}}\left|\boldsymbol{R}_{f}^{1} \eta\right|_{1}^{2}|\mathbf{v}|_{1}+\left.C_{\Omega_{f}}\left|\mathbf{f} \|_{0}\right| \mathbf{v}\right|_{1}}{|\mathbf{v}|_{1}} \\
& =C_{\mathcal{N}}\left|\boldsymbol{R}_{f}^{1} \eta\right|_{1}^{2}+C_{\Omega_{f}} \mid \mathbf{f} \|_{0} .
\end{aligned}
$$

Conditions $\alpha_{a}>0$ and

$$
C_{\mathcal{N}} \frac{\|\Pi \ell\|_{\left(V_{f}^{0}\right)^{\prime}}}{\alpha_{a}^{2}}<1
$$

are satisfied if

$$
C_{\mathcal{N}}\left|\boldsymbol{R}_{f}^{1} \eta\right|_{1}<\frac{1}{2} \frac{C_{\kappa} \nu}{2}
$$

and

$$
3\left(C_{\mathcal{N}}\left|\boldsymbol{R}_{f}^{1} \eta\right|_{1}\right)^{2}-4 \frac{C_{\kappa} \nu}{2} C_{\mathcal{N}}\left|\boldsymbol{R}_{f}^{1} \eta\right|_{1}+\left(\frac{C_{\kappa} \nu}{2}\right)^{2}-C_{\mathcal{N}} C_{\Omega_{f}}\|\mathbf{f}\|_{0}>0
$$

respectively. Condition (46) impose $\left(\frac{C_{\kappa} \nu}{2}\right)^{2}>C_{\mathcal{N}} C_{\Omega_{f}}\|\mathbf{f}\|_{0}$ in (47). This condition is (42), and, in this case, conditions (46) and (47) hold if (43) is satisfied.

4. Thanks to (43) and $1-3, a(\cdot ; \cdot, \cdot)$ and $\ell$ satisfy the hypotheses of Theorem 6.1 of Appendix, which allows us to conclude that there exists a unique solution $\mathcal{R}_{0}^{1}(\eta) \in V_{f}^{0}$ to $(38)$.

5. Since the inf-sup condition is satisfied, Theorem 6.2 guarantees that there exists a unique solution $\left(\mathcal{R}_{0}^{1}(\eta), \mathcal{R}_{0}^{2}(\eta)\right)$ to $(34)-(35)$. The thesis follows from (33) and from the uniqueness of the operator $R_{f}(26)$. 


\section{The interface equation associated to the cou- pled problem}

In this section we reformulate the global coupled problem (21)-(25) as an interface equation depending solely on $\lambda=\left(\mathbf{u}_{f} \cdot \mathbf{n}\right)_{\mid \Gamma}$.

We formally define the nonlinear pseudo-differential operator $\mathcal{S}: \Lambda_{0} \rightarrow \Lambda_{0}^{\prime}$,

$$
\begin{aligned}
\langle\mathcal{S}(\eta), \mu\rangle= & a_{f}\left(\boldsymbol{R}_{f}^{1}(\eta), \boldsymbol{R}^{1} \mu\right)+c_{f}\left(\boldsymbol{R}_{f}^{1}(\eta) ; \boldsymbol{R}_{f}^{1}(\eta), \boldsymbol{R}^{1} \mu\right)+b_{f}\left(\boldsymbol{R}^{1} \mu, \mathcal{R}_{f}^{2}(\eta)\right) \\
& +\int_{\Gamma} \sum_{j=1}^{d-1} \frac{\nu}{\varepsilon}\left(\boldsymbol{R}_{f}^{1}(\eta) \cdot \boldsymbol{\tau}_{j}\right)\left(\boldsymbol{R}^{1} \mu \cdot \boldsymbol{\tau}_{j}\right)-\int_{\Omega_{f}} \mathbf{f} \cdot\left(\boldsymbol{R}^{1} \mu\right) \\
& +\int_{\Gamma} g\left(R_{p} \eta\right) \mu \quad \forall \eta \in \Lambda_{0}, \forall \mu \in \Lambda .
\end{aligned}
$$

The operator $\mathcal{S}$ is composed of two parts: a non-linear component associated to the fluid problem in $\Omega_{f}$ (the terms in the first two lines), and a linear part related to the problem in the porous media (corresponding to the last integral). The fluid part plays the role of a non-linear Dirichlet-to-Neumann map that associates at any given normal velocity $\eta$ on $\Gamma$ the normal component of the corresponding Cauchy stress tensor on $\Gamma$. On the other hand, the linear porousmedia part is a Neumann-to-Dirichlet map that associates the trace on $\Gamma$ of the piezometric head whose conormal derivative on $\Gamma$ is equal to $\eta$.

We have the following equivalence result, whose proof follows the guidelines of Theorem 4.1 in [12].

Theorem 3.1 The solution of (21)-(25) can be characterized as follows:

$$
\mathbf{u}_{f}=\mathcal{R}_{f}^{1}(\lambda), \quad p_{f}=\mathcal{R}_{f}^{2}(\lambda)+\hat{p}_{f}, \quad \varphi=R_{p} \lambda,
$$

where $\hat{p}_{f}=\left(\operatorname{meas}\left(\Omega_{f}\right)\right)^{-1} \int_{\Omega_{f}} p_{f}$, and $\lambda \in \Lambda_{0}$ is the solution of the nonlinear interface problem:

$$
\langle\mathcal{S}(\lambda), \mu\rangle=0 \quad \forall \mu \in \Lambda_{0} .
$$

Moreover, $\hat{p}_{f}$ can be obtained from $\lambda$ by solving the algebraic equation

$$
\hat{p}_{f}=(\operatorname{meas}(\Gamma))^{-1}\langle\mathcal{S}(\lambda), \varepsilon\rangle,
$$

where $\varepsilon \in \Lambda$ is a fixed function such that

$$
\frac{1}{\operatorname{meas}(\Gamma)} \int_{\Gamma} \varepsilon=1
$$

Notice that a more useful characterization of the operator $\mathcal{S}$ can be provided. Indeed, with the special choice $\boldsymbol{R}^{1}=\boldsymbol{R}_{f}^{1}$ in (48), thanks to (27), we obtain

$$
b_{f}\left(\boldsymbol{R}_{f}^{1} \mu, \mathcal{R}_{f}^{2}(\eta)\right)=0 \quad \forall \eta, \mu \in \Lambda_{0} .
$$

Moreover, owing to (33), for $\eta, \mu \in \Lambda_{0}$, we have

$$
\begin{aligned}
\langle\mathcal{S}(\eta), \mu\rangle= & a_{f}\left(\boldsymbol{\mathcal { R }}_{0}^{1}(\eta)+\boldsymbol{R}_{f}^{1} \eta, \boldsymbol{R}_{f}^{1} \mu\right)+c_{f}\left(\boldsymbol{\mathcal { R }}_{0}^{1}(\eta)+\boldsymbol{R}_{f}^{1} \eta ; \boldsymbol{\mathcal { R }}_{0}^{1}(\eta)+\boldsymbol{R}_{f}^{1} \eta, \boldsymbol{R}_{f}^{1} \mu\right) \\
& +\int_{\Gamma} \sum_{j=1}^{d-1} \frac{\nu}{\varepsilon}\left(\left(\boldsymbol{\mathcal { R }}_{0}^{1}(\eta)+\boldsymbol{R}_{f}^{1} \eta\right) \cdot \boldsymbol{\tau}_{j}\right)\left(\boldsymbol{R}_{f}^{1} \mu \cdot \boldsymbol{\tau}_{j}\right) \\
& -\int_{\Omega_{f}} \mathbf{f} \cdot\left(\boldsymbol{R}_{f}^{1} \mu\right)+\int_{\Gamma} g\left(R_{p} \eta\right) \mu .
\end{aligned}
$$


By taking $\mathcal{R}_{0}^{1}(\eta) \in H_{f}^{0}$ as test function in (27), we obtain:

$$
a_{f}\left(\boldsymbol{R}_{f}^{1} \mu, \boldsymbol{\mathcal { R }}_{0}^{1}(\eta)\right)+b_{f}\left(\boldsymbol{\mathcal { R }}_{0}^{1}(\eta), R_{f}^{2} \mu\right)+\int_{\Gamma} \sum_{j=1}^{d-1} \frac{\nu}{\varepsilon}\left(\boldsymbol{R}_{f}^{1} \mu \cdot \boldsymbol{\tau}_{j}\right)\left(\boldsymbol{\mathcal { R }}_{0}^{1}(\eta) \cdot \boldsymbol{\tau}_{j}\right)=0
$$

Finally, since $R_{f}^{2} \mu \in Q_{0}$, owing to (34)-(35) it follows that

$$
a_{f}\left(\boldsymbol{R}_{f}^{1} \mu, \boldsymbol{\mathcal { R }}_{0}^{1}(\eta)\right)+\int_{\Gamma} \sum_{j=1}^{d-1} \frac{\nu}{\varepsilon}\left(\boldsymbol{R}_{f}^{1} \mu \cdot \boldsymbol{\tau}_{j}\right)\left(\boldsymbol{\mathcal { R }}_{0}^{1}(\eta) \cdot \boldsymbol{\tau}_{j}\right)=0,
$$

so that, for all $\eta, \mu \in \Lambda_{0}$, the operator $\mathcal{S}$ can be characterized as

$$
\begin{aligned}
\langle\mathcal{S}(\eta), \mu\rangle= & a_{f}\left(\boldsymbol{R}_{f}^{1} \eta, \boldsymbol{R}_{f}^{1} \mu\right)+c_{f}\left(\boldsymbol{\mathcal { R }}_{0}^{1}(\eta)+\boldsymbol{R}_{f}^{1} \eta ; \boldsymbol{\mathcal { R }}_{0}^{1}(\eta)+\boldsymbol{R}_{f}^{1} \eta, \boldsymbol{R}_{f}^{1} \mu\right) \\
& +\int_{\Gamma} \sum_{j=1}^{d-1} \frac{\nu}{\varepsilon}\left(\boldsymbol{R}_{f}^{1} \eta \cdot \boldsymbol{\tau}_{j}\right)\left(\boldsymbol{R}_{f}^{1} \mu \cdot \boldsymbol{\tau}_{j}\right) \\
& -\int_{\Omega_{f}} \mathbf{f} \cdot\left(\boldsymbol{R}_{f}^{1} \mu\right)+\int_{\Gamma} g\left(R_{p} \eta\right) \mu .
\end{aligned}
$$

We study now the well-posedness of the nonlinear interface problem (50).

Note that in view of $(52), \mathcal{S}(\lambda)$ is defined in terms of the operator $\boldsymbol{\mathcal { R }}_{0}^{1}(\lambda)$, which, thanks to (34)-(35), satisfies in its turn the following problem:

$$
\begin{aligned}
a_{f}\left(\boldsymbol{\mathcal { R }}_{0}^{1}(\lambda), \mathbf{v}\right)+ & c_{f}\left(\boldsymbol{\mathcal { R }}_{0}^{1}(\lambda)+\boldsymbol{R}_{f}^{1} \lambda ; \boldsymbol{\mathcal { R }}_{0}^{1}(\lambda)+\boldsymbol{R}_{f}^{1} \lambda, \mathbf{v}\right) \\
& +\int_{\Gamma} \sum_{j=1}^{d-1} \frac{\nu}{\varepsilon}\left(\boldsymbol{\mathcal { R }}_{0}^{1}(\lambda) \cdot \boldsymbol{\tau}_{j}\right)\left(\mathbf{v} \cdot \boldsymbol{\tau}_{j}\right)=\int_{\Omega_{f}} \mathbf{f} \cdot \mathbf{v} \quad \forall \mathbf{v} \in V_{f}^{0}
\end{aligned}
$$

Therefore, in order to prove the existence and uniqueness of the solution of the interface problem, we have to consider (50), with the characterization of $\mathcal{S}$ given in (52), coupled with (53), i.e., we have to guarantee at once the existence and uniqueness of $\lambda \in \Lambda_{0}$ and $\mathcal{R}_{0}^{1}(\lambda) \in V_{f}^{0}$. To this aim we apply Theorem 6.1 considering the product space $W=\Lambda_{0} \times V_{f}^{0}$ endowed with the norm:

$$
\|\bar{v}\|_{W}=\left(\left|\boldsymbol{R}_{f}^{1} \mu\right|_{1}^{2}+|\mathbf{v}|_{1}^{2}\right)^{1 / 2} \quad \forall \bar{v}=(\mu, \mathbf{v}) \in W .
$$

We introduce the trilinear form and the linear functional associated with our problem in the space $W$. For any fixed $(\eta, \mathbf{w}) \in W$, we define the following operator depending on $\bar{w}$ :

$$
\begin{aligned}
\tilde{\mathcal{A}}(\eta, \mathbf{w}): W & \rightarrow W^{\prime} \\
\langle(\tilde{\mathcal{A}}(\eta, \mathbf{w}))(\xi, \mathbf{u}),(\mu, \mathbf{v})\rangle & =\left\langle\left(\mathcal{A}_{f}(\eta, \mathbf{w})\right)(\xi, \mathbf{u}), \mu\right\rangle+\left\langle\left(\mathcal{A}_{0}(\eta, \mathbf{w})\right)(\xi, \mathbf{u}), \mathbf{v}\right\rangle
\end{aligned}
$$

where, for every test function $\mu \in \Lambda_{0}$,

$$
\begin{aligned}
\left\langle\left(\mathcal{A}_{f}(\eta, \mathbf{w})\right)(\xi, \mathbf{u}), \mu\right\rangle= & a_{f}\left(\boldsymbol{R}_{f}^{1} \xi, \boldsymbol{R}_{f}^{1} \mu\right)+c_{f}\left(\mathbf{w}+\boldsymbol{R}_{f}^{1} \eta ; \mathbf{u}+\boldsymbol{R}_{f}^{1} \xi, \boldsymbol{R}_{f}^{1} \mu\right) \\
& +\int_{\Gamma} \sum_{j=1}^{d-1} \frac{\nu}{\varepsilon}\left(\boldsymbol{R}_{f}^{1} \xi \cdot \boldsymbol{\tau}_{j}\right)\left(\boldsymbol{R}_{f}^{1} \mu \cdot \boldsymbol{\tau}_{j}\right)+\int_{\Gamma} g\left(R_{p} \xi\right) \mu,
\end{aligned}
$$


whereas for any test function $\mathbf{v} \in V_{f}^{0}$,

$$
\begin{aligned}
\left\langle\left(\mathcal{A}_{0}(\eta, \mathbf{w})\right)(\xi, \mathbf{u}), \mathbf{v}\right\rangle= & a_{f}(\mathbf{u}, \mathbf{v})+c_{f}\left(\mathbf{w}+\boldsymbol{R}_{f}^{1} \eta ; \mathbf{u}+\boldsymbol{R}_{f}^{1} \xi, \mathbf{v}\right) \\
& +\int_{\Gamma} \sum_{j=1}^{d-1} \frac{\nu}{\varepsilon}\left(\mathbf{u} \cdot \boldsymbol{\tau}_{j}\right)\left(\mathbf{v} \cdot \boldsymbol{\tau}_{j}\right)
\end{aligned}
$$

We indicate by $\tilde{a}$ the form associated to the operator $\tilde{\mathcal{A}}$ :

$$
\tilde{a}(\bar{w} ; \bar{u}, \bar{v})=\langle(\tilde{\mathcal{A}}(\eta, \mathbf{w}))(\xi, \mathbf{u}),(\mu, \mathbf{v})\rangle
$$

for all $\bar{w}=(\eta, \mathbf{w}), \bar{u}=(\xi, \mathbf{u}), \bar{v}=(\mu, \mathbf{v}) \in W$.

Next, we define two functionals $\ell_{f}: \Lambda_{0} \rightarrow \mathbb{R}$ and $\ell_{0}: V_{f}^{0} \rightarrow \mathbb{R}$ as:

$$
\begin{aligned}
& \left\langle\ell_{f}, \mu\right\rangle=\int_{\Omega_{f}} \mathbf{f} \cdot\left(\boldsymbol{R}_{f}^{1} \mu\right) \quad \forall \mu \in \Lambda_{0}, \\
& \left\langle\ell_{0}, \mathbf{v}\right\rangle=\int_{\Omega_{f}} \mathbf{f} \cdot \mathbf{v} \quad \forall \mathbf{v} \in V_{f}^{0},
\end{aligned}
$$

and denote

$$
\langle\tilde{\ell}, \bar{v}\rangle=\left\langle\ell_{f}, \mu\right\rangle+\left\langle\ell_{0}, \mathbf{v}\right\rangle \quad \forall \bar{v}=(\mu, \mathbf{v}) \in W .
$$

Thus, the problem defined by (50) and (53) can be reformulated as:

$$
\text { find } \bar{u}=(\lambda, u) \in W: \quad \tilde{a}(\bar{u} ; \bar{u}, \bar{v})=\langle\tilde{\ell}, \bar{v}\rangle \quad \forall \bar{v}=(\mu, \mathbf{v}) \in W .
$$

We shall prove the existence and uniqueness of the solution only in a closed convex subset of $W$.

Lemma 3.1 Let $\mathbf{f} \in L^{2}\left(\Omega_{f}\right)$ be such that

$$
2(1+\sqrt{2}) \sqrt{2 C_{\mathcal{N}} C_{\Omega_{f}}\|\mathbf{f}\|_{0}} \leq C_{\kappa} \nu,
$$

and consider two constants

$$
r_{m}=\frac{C_{1}-\sqrt{C_{1}^{2}-4 C_{2}}}{2} \text { and } r_{M}=C_{1}-\sqrt{\sqrt{2} C_{2}},
$$

where

$$
C_{1}=\frac{C_{\kappa} \nu}{4 C_{\mathcal{N}}}, \quad C_{2}=\frac{\sqrt{2} C_{\Omega_{f}}\|\mathbf{f}\|_{0}}{2 C_{\mathcal{N}}} .
$$

Notice that, thanks to (58), there holds

$$
0 \leq r_{m}<r_{M} .
$$

If we consider

$$
\bar{B}_{r}=\left\{\bar{w}=(\eta, \mathbf{w}) \in W:\left|\boldsymbol{R}_{f}^{1} \eta\right|_{1} \leq r\right\},
$$

with

$$
r_{m}<r<r_{M},
$$

then, there exists a unique solution $\bar{u}=(\lambda, u) \in \bar{B}_{r}$ of $(57)$ with $u=\mathcal{R}_{0}^{1}(\lambda)$. 
Remark 3.1 Condition (58) is equivalent to

$$
C_{1}^{2} \geq \frac{3+2 \sqrt{2}}{\sqrt{2}} C_{2} .
$$

Proof. The proof is composed of several parts.

1. For each $\bar{w}=(\eta, \mathbf{w}) \in \bar{B}_{r}$ the bilinear form $\tilde{a}(\bar{w} ; \cdot, \cdot)$ is uniformly coercive on $W$.

By definition, for all $\bar{v}=(\mu, \mathbf{v}) \in W$ we have

$$
\begin{aligned}
\tilde{a}(\bar{w} ; \bar{v}, \bar{v})= & a_{f}\left(\boldsymbol{R}_{f}^{1} \mu, \boldsymbol{R}_{f}^{1} \mu\right)+a_{f}(\mathbf{v}, \mathbf{v})+\int_{\Gamma} g\left(R_{p} \mu\right) \mu \\
& +c_{f}\left(\mathbf{w}+\boldsymbol{R}_{f}^{1} \eta ; \mathbf{v}+\boldsymbol{R}_{f}^{1} \mu, \mathbf{v}+\boldsymbol{R}_{f}^{1} \mu\right) \\
& +\int_{\Gamma} \sum_{j=1}^{d-1} \frac{\nu}{\varepsilon}\left(\boldsymbol{R}_{f}^{1} \mu \cdot \boldsymbol{\tau}_{j}\right)\left(\boldsymbol{R}_{f}^{1} \mu \cdot \boldsymbol{\tau}_{j}\right) \\
& +\int_{\Gamma} \sum_{j=1}^{d-1} \frac{\nu}{\varepsilon}\left(\mathbf{v} \cdot \boldsymbol{\tau}_{j}\right)\left(\mathbf{v} \cdot \boldsymbol{\tau}_{j}\right) .
\end{aligned}
$$

Thanks to (30), we have $\int_{\Gamma} g\left(R_{p} \mu\right) \mu \geq 0$. Using the inequalities (40) and (41) and the fact that $\mathbf{w} \in V_{f}^{0}$, we obtain

$$
\tilde{a}(\bar{w} ; \bar{v}, \bar{v}) \geq \frac{C_{k} \nu}{2}\left(\left|\boldsymbol{R}_{f}^{1} \mu\right|_{1}^{2}+|\mathbf{v}|_{1}^{2}\right)-2 C_{\mathcal{N}}\left|\boldsymbol{R}_{f}^{1} \eta\right|_{1}\left(\left|\boldsymbol{R}_{f}^{1} \mu\right|_{1}^{2}+|\mathbf{v}|_{1}^{2}\right)
$$

Thus,

$$
\tilde{a}(\bar{w} ; \bar{v}, \bar{v}) \geq \alpha_{\tilde{a}}\left(\left|\boldsymbol{R}_{f}^{1} \mu\right|_{1}^{2}+|\mathbf{v}|_{1}^{2}\right)
$$

having set

$$
\alpha_{\tilde{a}}=\frac{C_{k} \nu}{2}-2 C_{\mathcal{N}}\left|\boldsymbol{R}_{f}^{1} \eta\right|_{1}
$$

Condition $\alpha_{\tilde{a}}>0$ is equivalent to $\left|\boldsymbol{R}_{f}^{1} \eta\right|_{1}<C_{1}$, which is satisfied in view of (61), (59) and (63). Thus, the bilinear form $\tilde{a}(\bar{w} ; \cdot, \cdot)$ is uniformly coercive with respect to any $\bar{w} \in \bar{B}_{r}$.

Thanks to the Lax-Milgram Lemma (see, e.g., [25] p. 133) the operator $\tilde{\mathcal{A}}(\bar{w}) \in$ $\mathcal{L}\left(W ; W^{\prime}\right)$ is invertible for each $\bar{w} \in \bar{B}_{r}$. Moreover, the inverse $\mathcal{T}(\bar{w})=(\tilde{\mathcal{A}}(\bar{w}))^{-1}$ belongs to $\mathcal{L}\left(W^{\prime} ; W\right)$ and it satisfies

$$
\|\mathcal{T}(\bar{w})\|_{\mathcal{L}\left(W^{\prime} ; W\right)} \leq \frac{1}{\alpha_{\tilde{a}}}
$$

Now, we prove that there exists a unique $\bar{u} \in \bar{B}_{r}$ such that $\bar{u}=\mathcal{T}(\bar{u}) \tilde{\ell}$, i.e., $(57)$ has a unique solution in $\bar{B}_{r}$.

2. $\bar{v} \rightarrow \mathcal{T}(\bar{v}) \tilde{\ell}$ maps $\bar{B}_{r}$ into $\bar{B}_{r}$ and is a strict contraction in $\bar{B}_{r}$.

For all $\bar{v}=(\mu, \mathbf{v}) \in \bar{B}_{r}$ we have

$$
\|\mathcal{T}(\bar{v}) \tilde{\ell}\|_{W} \leq\|\mathcal{T}(\bar{v})\|_{\mathcal{L}\left(W^{\prime} ; W\right)}\|\tilde{\ell}\|_{W^{\prime}} \leq \frac{\|\tilde{\ell}\|_{W^{\prime}}}{\alpha_{\tilde{a}}}
$$


Moreover,

$$
\begin{aligned}
\|\tilde{\ell}\|_{W^{\prime}} & =\sup _{\bar{v} \in W, \bar{v} \neq 0} \frac{\left|\int_{\Omega_{f}} \mathbf{f} \cdot\left(\boldsymbol{R}_{f}^{1} \mu\right)+\int_{\Omega_{f}} \mathbf{f} \cdot \mathbf{v}\right|}{\|\bar{v}\|_{W}} \\
& \leq C_{\Omega_{f}}\|\mathbf{f}\|_{0} \sup _{\bar{v} \in W, \bar{v} \neq 0} \frac{\left|\boldsymbol{R}_{f}^{1} \mu\right|_{1}+|\mathbf{v}|_{1}}{\|\bar{v}\|_{W}} \leq \sqrt{2} C_{\Omega_{f}}\|\mathbf{f}\|_{0} .
\end{aligned}
$$

From (67) and (66), corresponding to some $\bar{w}=(\eta, \mathbf{w}) \in \bar{B}_{r}$, condition

$$
\frac{\|\tilde{\ell}\|_{W^{\prime}}}{\alpha_{\tilde{a}}} \leq r
$$

is satisfied if

$$
r^{2}-C_{1} r+C_{2} \leq 0
$$

that is $r_{\min } \leq r \leq r_{\max }$ with

$$
r_{\min }=\frac{C_{1}-\sqrt{C_{1}^{2}-4 C_{2}}}{2} \quad \text { and } \quad r_{\max }=\frac{C_{1}+\sqrt{C_{1}^{2}-4 C_{2}}}{2} .
$$

Since $C_{1}^{2}-4 C_{2} \geq 0$ from (64), for any $\bar{v} \in \bar{B}_{r}$ with $r$ satisfying $(68), \mathcal{T}(\bar{v}) \tilde{\ell}$ belongs to $\bar{B}_{r}$.

Finally, to find $r$ such that that the map $\bar{v} \rightarrow \mathcal{T}(\bar{v}) \tilde{\ell}$ is a strict contraction in $\bar{B}_{r}$, we should guarantee (see [17] p. 282) that for any $\bar{w}_{1}, \bar{w}_{2} \in \bar{B}_{r}$

$$
\left\|\left(\mathcal{T}\left(\bar{w}_{1}\right)-\mathcal{T}\left(\bar{w}_{2}\right)\right) \tilde{\ell}\right\|_{W} \leq \frac{\|\tilde{\ell}\|_{W^{\prime}}}{\alpha_{\tilde{a}}^{2}} L(r)\left\|\bar{w}_{1}-\bar{w}_{2}\right\|_{W}<\left\|\bar{w}_{1}-\bar{w}_{2}\right\|_{W}
$$

$L(r)$ being the Lipschitz continuity constant associated to $\tilde{\mathcal{A}}$. However,

$$
\begin{aligned}
\mid\left\langle\left(\tilde{\mathcal{A}}\left(\bar{w}_{1}\right)\right.\right. & \left.\left.-\tilde{\mathcal{A}}\left(\bar{w}_{2}\right)\right)(\bar{u}), \bar{v}\right\rangle|=| \tilde{a}\left(\bar{w}_{1} ; \bar{u}, \bar{v}\right)-\tilde{a}\left(\bar{w}_{2} ; \bar{u}, \bar{v}\right) \mid \\
& =\left|c_{f}\left(\mathbf{w}_{1}+\boldsymbol{R}_{f}^{1} \eta_{1}-\left(\mathbf{w}_{2}+\boldsymbol{R}_{f}^{1} \eta_{2}\right) ; \mathbf{u}+\boldsymbol{R}_{f}^{1} \lambda, \mathbf{v}+\boldsymbol{R}_{f}^{1} \mu\right)\right| \\
& \leq C_{\mathcal{N}}\left|\mathbf{w}_{1}+\boldsymbol{R}_{f}^{1} \eta_{1}-\mathbf{w}_{2}-\boldsymbol{R}_{f}^{1} \eta_{2}\right|_{1}\left|\mathbf{u}+\boldsymbol{R}_{f}^{1} \lambda\right|_{1}\left|\mathbf{v}+\boldsymbol{R}_{f}^{1} \mu\right|_{1} \\
& \leq 2 \sqrt{2} C_{\mathcal{N}}\left\|\bar{w}_{1}-\bar{w}_{2}\right\|_{W}\|\bar{u}\|_{W}\|\bar{v}\|_{W}
\end{aligned}
$$

so that $L(r)=2 \sqrt{2} C_{\mathcal{N}}$. Thus, condition

$$
\frac{\|\tilde{\ell}\|_{W^{\prime}}}{\alpha_{\tilde{a}}^{2}} L(r)<1
$$

is satisfied if

$$
r^{2}-2 C_{1} r+C_{1}^{2}-\sqrt{2} C_{2}>0
$$

i.e.,

$$
r<r_{M I N}=C_{1}-\sqrt{\sqrt{2} C_{2}} \quad \text { or } \quad r>r_{M A X}=C_{1}+\sqrt{\sqrt{2} C_{2}} .
$$

It is easy to see that $r_{\max }<r_{M A X}$. Consequently, there exists a $r$ which satisfies (68) and (70) if and only if $r_{\min } \leq r_{M I N}$, which is equivalent to condition (64) 
or to condition (58). Under this condition, any $r$ in the interval (63) with $r_{m}=r_{\min }$ and $r_{M}=r_{M I N}$, will satisfy both (68) and (70).

3. The existence and uniqueness of the solution $\bar{u}=\left(\lambda, \boldsymbol{\mathcal { R }}_{0}^{1}(\lambda)\right) \in \bar{B}_{r}$ to (57) is now a simple consequence of the Banach contraction theorem (see, e.g., [27]).

The following theorem is a direct consequence of the previous lemma.

Theorem 3.2 If (58) holds, then problem (57) has a unique solution $\bar{u}=$ $\left(\lambda, \mathcal{R}_{0}^{1}(\lambda)\right)$ in the set

$$
B_{r_{M}}=\left\{\bar{w}=(\eta, \mathbf{w}) \in W:\left|\boldsymbol{R}_{f}^{1} \eta\right|_{1}<r_{M}\right\},
$$

and it satisfies $\left|\boldsymbol{R}_{f}^{1} \lambda\right|_{1} \leq r_{m}$, where $r_{m}$ and $r_{M}$ are defined in (59). In particular, it follows that (50) has a unique solution $\lambda$ in the set $S_{r_{M}}=\left\{\eta \in \Lambda_{0}:\left|\boldsymbol{R}_{f}^{1} \eta\right|_{1}<\right.$ $\left.r_{M}\right\} \subset \Lambda_{0}$ which indeed belongs to $S_{r_{m}}=\left\{\eta \in \Lambda_{0}:\left|\boldsymbol{R}_{f}^{1} \eta\right|_{1} \leq r_{m}\right\}$.

Proof. Since problem (50) has a solution $\lambda$ if and only if $\bar{u}=\left(\lambda, \boldsymbol{\mathcal { R }}_{0}^{1}(\lambda)\right)$ is a solution of problem (57), we prove only the first part of theorem.

From the previous Lemma 3.1, if (58) holds, (57) has at least a solution in $B_{r_{M}}$ as it has a solution in $\bar{B}_{r} \subset B_{r_{M}}$, for any $r_{m}<r<r_{M}$. To prove the uniqueness, let us assume that (57) has two solutions $\bar{u}_{1}=\left(\lambda_{1},\left(\mathcal{R}_{0}^{1}(\lambda)\right)_{1}\right) \neq$ $\bar{u}_{2}=\left(\lambda_{2},\left(\boldsymbol{R}_{0}^{1}(\lambda)\right)_{2}\right)$ in $B_{r_{M}}$. Then, $r_{1}=\left|\boldsymbol{R}_{f}^{1} \lambda_{1}\right|_{1}<r_{M}$ and $r_{2}=\left|\boldsymbol{R}_{f}^{1} \lambda_{2}\right|_{1}<$ $r_{M}$. Therefore, any set $\bar{B}_{r}$ with $\max \left\{r_{m}, r_{1}, r_{2}\right\}<r<r_{M}$ contains two different solutions of problem (57). This contradicts the result of Lemma 3.1. Now, let $\bar{u}=\left(\lambda, \mathcal{R}_{0}^{1}(\lambda)\right)$ be the unique solution of problem (57) in $B_{r_{M}}$. According to Lemma 3.1, it belongs to each $\bar{B}_{r} \subset B_{r_{M}}$ with $r_{m}<r<r_{M}$, and consequently $\left|\boldsymbol{R}_{f}^{1} \lambda\right|_{1} \leq r_{m}$.

Remark 3.2 Notice that condition (58) is analogous to that usually required to prove existence and uniqueness of the solution of the Navier-Stokes equations. Moreover, we have proved that the solution is unique in $S_{r_{M}}$. Thus, in view of Remark 2.1, Theorem 3.2 states that the solution is unique only for sufficiently small normal velocities $\lambda$ across the interface $\Gamma$. Finally, notice that (58) implies (42) and that $S_{r_{m}}$ is included in the set (43), so that the existence and uniqueness of the nonlinear extension $\mathcal{R}_{0}^{1}(\lambda)$ is ensured as well.

\section{Iterative finite element solution of the coupled problem}

In this section, we introduce and analyze some iterative methods to compute the solution of a conforming finite element approximation of (16)-(18). For the easiness of notation, we will write the algorithms in continuous form. However, they can be straightforwardly translated into a discrete setting considering conforming internal Galerkin approximations of the spaces (7)-(11).

Moreover, the convergence results that we will present hold in the discrete case without any dependence of the convergence rate on the grid parameter $h$, since they are established by using the properties of the operators in the continuous case. 
As concerns the finite element approximation, let us just indicate our basic assumptions, referring to $[13,10,14]$ for a more detailed description.

We consider a regular triangulation of the domain $\bar{\Omega}_{f} \cup \bar{\Omega}_{p}$, depending on a positive parameter $h>0$, made up of triangles if $d=2$, or tetrahedra in the 3 dimensional case. We assume that the triangulations induced on the subdomains $\Omega_{f}$ and $\Omega_{p}$ are compatible on $\Gamma$, that is they share the same edges (if $d=2$ ) or faces (if $d=3$ ) therein. Finally we suppose the triangulation induced on $\Gamma$ to be quasi-uniform (see, e.g., [25]).

Several choices of finite element spaces can be made. However, in order to guarantee the stability of the approximation of the coupled problem, we must only ensure that the finite element spaces which approximate the velocity and pressure fields in the fluid domain, say $H_{f h}$ and $Q_{h}$, respectively, satisfy an infsup condition. Indeed, there must exist a positive constant $\beta^{*}>0$, independent of $h$, such that $\forall q_{h} \in Q_{h}, \exists \mathbf{v}_{h} \in H_{f h}, \mathbf{v}_{h} \neq \mathbf{0}$ such that

$$
\int_{\Omega_{f}} q_{h} \nabla \cdot \mathbf{v}_{h} \geq \beta^{*}\left\|\mathbf{v}_{h}\right\|_{1}\left\|q_{h}\right\|_{0}
$$

No additional condition is required on the discrete space used to approximate the piezometric head in $\Omega_{p}$.

Several families of finite element spaces satisfying the inf-sup condition (71) are provided in [4]. The classical error estimates hold for the Navier-Stokes equations (see, e.g., [17, Chapter II]) and for the Darcy problem (6) (see, e.g., [25]). In our numerical results we will make the special choice of piecewise quadratic elements for the velocity components and piecewise linear for the pressure (the so-called Taylor-Hood elements), while we will use piecewise quadratic elements for approximating the piezometric head $\varphi$ in $\Omega_{p}$. For such choices, it is wellknown that the following error estimates hold: there exist two positive constants $C_{1}$ and $C_{2}$ such that:

$$
E_{N S}^{h} \leq C_{1} h^{r}\left(\left\|\mathbf{u}_{f}\right\|_{r+1}+\left\|p_{f}\right\|_{r}\right), \quad r=1,2,
$$

if $\mathbf{u}_{f} \in H^{r+1}\left(\Omega_{f}\right)$ and $p_{f} \in H^{r}\left(\Omega_{f}\right)$, where

$$
E_{N S}^{h}=\left\|\nabla \mathbf{u}_{f}-\nabla \mathbf{u}_{f h}\right\|_{0}+\left\|p_{f}-p_{f h}\right\|_{0},
$$

while

$$
E_{D}^{h} \leq C_{2} h^{l}\|\varphi\|_{l+1}, \quad l=\min (2, s-1),
$$

if $\varphi \in H^{s}\left(\Omega_{p}\right), s \geq 2$, with

$$
E_{D}^{h}=\left\|\varphi-\varphi_{h}\right\|_{1}
$$

We have indicated by the subscript $h$ the finite element approximations of $\mathbf{u}_{f}$, $p_{f}$ and $\varphi$. We will verify these estimates numerically in Sect. 5 . 


\subsection{Fixed-point iterations}

Fixed-point iterations to solve the coupled problem (16)-(18) can be written as follows. Given $\mathbf{u}_{f}^{0} \in H_{f}$, for $n \geq 1$, find $\mathbf{u}_{f}^{n} \in H_{f}, p_{f}^{n} \in Q, \varphi^{n} \in H_{p}$ such that

$$
\begin{aligned}
& a_{f}\left(\mathbf{u}_{f}^{n}, \mathbf{v}\right)+c_{f}\left(\mathbf{u}_{f}^{n-1} ; \mathbf{u}_{f}^{n}, \mathbf{v}\right)+b_{f}\left(\mathbf{v}, p_{f}^{n}\right) \\
& +\int_{\Gamma} g \varphi^{n}(\mathbf{v} \cdot \mathbf{n})+\int_{\Gamma} \sum_{j=1}^{d-1} \frac{\nu}{\varepsilon}\left(\mathbf{u}_{f}^{n} \cdot \boldsymbol{\tau}_{j}\right)\left(\mathbf{v} \cdot \boldsymbol{\tau}_{j}\right)=\int_{\Omega_{f}} \mathbf{f} \cdot \mathbf{v}, \\
& b_{f}\left(\mathbf{u}_{f}^{n}, q\right)=0 \\
& a_{p}\left(\varphi^{n}, \psi\right)=\int_{\Gamma} \psi\left(\mathbf{u}_{f}^{n} \cdot \mathbf{n}\right)
\end{aligned}
$$

for all $\mathbf{v} \in H_{f}, q \in Q, \psi \in H_{p}$.

Algorithm (74)-(76) requires to solve at each iteration a linear coupled problem, and it can be reinterpreted as a fixed-point method to solve the interface problem (57). Indeed, let us first rewrite (74)-(75) in the equivalent form:

find $\mathbf{u}_{f}^{n} \in V_{f}$ such that

$$
\begin{aligned}
a_{f}\left(\mathbf{u}_{f}^{n}, \mathbf{v}\right)+c_{f}\left(\mathbf{u}_{f}^{n-1}\right. & \left.; \mathbf{u}_{f}^{n}, \mathbf{v}\right)+\int_{\Gamma} g \varphi^{n}(\mathbf{v} \cdot \mathbf{n}) \\
& +\int_{\Gamma} \sum_{j=1}^{d-1} \frac{\nu}{\varepsilon}\left(\mathbf{u}_{f}^{n} \cdot \boldsymbol{\tau}_{j}\right)\left(\mathbf{v} \cdot \boldsymbol{\tau}_{j}\right)=\int_{\Omega_{f}} \mathbf{f} \cdot \mathbf{v} \quad \forall \mathbf{v} \in V_{f} .
\end{aligned}
$$

We denote $\lambda^{n}=\mathbf{u}_{f}^{n} \cdot \mathbf{n}$ on $\Gamma$ and we remark that $\lambda^{n} \in \Lambda_{0}$. Then, we consider $\boldsymbol{R}_{f}^{1} \lambda^{n} \in H_{f}$ and we set $\mathbf{u}^{n}=\mathbf{u}_{f}^{n}-\boldsymbol{R}_{f}^{1} \lambda^{n}$. By definition, $\mathbf{u}^{n} \cdot \mathbf{n}=0$ on $\Gamma$. Moreover, remark that for all $\eta \in \Lambda_{0},(28)$ implies $\nabla \cdot \boldsymbol{R}_{f}^{1} \eta=0$ in $\Omega_{f}$ since we can choose $q=\nabla \cdot \boldsymbol{R}_{f}^{1} \eta$. Thus, $\mathbf{u}^{n} \in V_{f}^{0}$.

From (76) it follows $-\mathrm{K} \nabla \varphi^{n} \cdot \mathbf{n}=\mathbf{u}_{f}^{n} \cdot \mathbf{n}=\lambda^{n}$ on $\Gamma$, so that by definition of $R_{p}$, we can write $\varphi^{n}=R_{p} \lambda^{n}$.

Finally, since $\mathbf{v} \in V_{f}$, proceeding as for $\mathbf{u}_{f}^{n}$, we can split $\mathbf{v}=\mathbf{w}+\boldsymbol{R}_{f}^{1} \mu$ with $\mu=\mathbf{v} \cdot \mathbf{n}$ on $\Gamma$ and $\mathbf{w} \in V_{f}^{0}$. Thus, (77) becomes

$$
\begin{array}{r}
a_{f}\left(\mathbf{u}^{n}+\boldsymbol{R}_{f}^{1} \lambda^{n}, \mathbf{w}+\boldsymbol{R}_{f}^{1} \mu\right)+c_{f}\left(\mathbf{u}^{n-1}+\boldsymbol{R}_{f}^{1} \lambda^{n-1} ; \mathbf{u}^{n}+\boldsymbol{R}_{f}^{1} \lambda^{n}, \mathbf{w}+\boldsymbol{R}_{f}^{1} \mu\right) \\
+\int_{\Gamma} g\left(R_{p} \lambda^{n}\right)\left(\mathbf{w}+\boldsymbol{R}_{f}^{1} \mu\right) \cdot \mathbf{n}+\int_{\Gamma} \sum_{j=1}^{d-1} \frac{\nu}{\varepsilon}\left(\left(\mathbf{u}^{n}+\boldsymbol{R}_{f}^{1} \lambda^{n}\right) \cdot \boldsymbol{\tau}_{j}\right)\left(\left(\mathbf{w}+\boldsymbol{R}_{f}^{1} \mu\right) \cdot \boldsymbol{\tau}_{j}\right) \\
=\int_{\Omega_{f}} \mathbf{f} \cdot\left(\mathbf{w}+\boldsymbol{R}_{f}^{1} \mu\right) .
\end{array}
$$

Taking into account that $\mathbf{w} \in V_{f}^{0}$ and the definition of $\boldsymbol{R}_{f}^{1}(27)-(28)$, this corresponds to the fixed-point method: given $\bar{u}^{n-1}=\left(\lambda^{n-1}, \mathbf{u}^{n-1}\right) \in W$, for $n \geq 1$,

$$
\text { find } \bar{u}^{n}=\left(\lambda^{n}, \mathbf{u}^{n}\right) \in W: \quad \tilde{a}\left(\bar{u}^{n-1} ; \bar{u}^{n}, \bar{w}\right)=\langle\tilde{\ell}, \bar{w}\rangle \quad \forall \bar{w}=(\mu, \mathbf{w}) \in W .
$$

Then, in view of this equivalence, the convergence of (74)-(76) is a direct consequence of Lemma 3.1. We can state the following result which is a straightforward corollary of Theorem 3.2. 
Proposition 4.1 If (58) holds and if $\mathbf{u}_{f}^{0}$ is such that $\left|\boldsymbol{R}_{f}^{1}\left(\mathbf{u}_{f}^{0} \cdot \mathbf{n}\right)\right|_{1}<r_{M}$ with $r_{M}$ given in (59), then the sequence $\left(\mathbf{u}_{f}^{n}, p_{f}^{n}, \varphi^{n}\right)$ converges for $n \rightarrow \infty$ to the unique solution $\left(\mathbf{u}_{f}, p_{f}, \varphi\right)$ of problem (16)-(18), and $\left|\boldsymbol{R}_{f}^{1}\left(\mathbf{u}_{f} \cdot \mathbf{n}\right)\right|_{1} \leq r_{m}$.

\subsection{Newton-like methods}

Let us consider now the Newton method to solve (the discrete form of) (16)(18).

Let $\mathbf{u}_{f}^{0} \in H_{f}$ be given. Then, for $n \geq 1$, the Newton method reads: find $\mathbf{u}_{f}^{n} \in H_{f}, p_{f}^{n} \in Q, \varphi^{n} \in H_{p}$ such that

$$
\begin{aligned}
& a_{f}\left(\mathbf{u}_{f}^{n}, \mathbf{v}\right)+c_{f}\left(\mathbf{u}_{f}^{n} ; \mathbf{u}_{f}^{n-1}, \mathbf{v}\right)+c_{f}\left(\mathbf{u}_{f}^{n-1} ; \mathbf{u}_{f}^{n}, \mathbf{v}\right)+b_{f}\left(\mathbf{v}, p_{f}^{n}\right)+\int_{\Gamma} g \varphi^{n}(\mathbf{v} \cdot \mathbf{n}) \\
& \quad+\int_{\Gamma} \sum_{j=1}^{d-1} \frac{\nu}{\varepsilon}\left(\mathbf{u}_{f}^{n} \cdot \boldsymbol{\tau}_{j}\right)\left(\mathbf{v} \cdot \boldsymbol{\tau}_{j}\right)=c_{f}\left(\mathbf{u}_{f}^{n-1} ; \mathbf{u}_{f}^{n-1}, \mathbf{v}\right)+\int_{\Omega_{f}} \mathbf{f} \cdot \mathbf{v}, \\
& b_{f}\left(\mathbf{u}_{f}^{n}, q\right)=0, \\
& a_{p}\left(\varphi^{n}, \psi\right)=\int_{\Gamma} \psi\left(\mathbf{u}_{f}^{n} \cdot \mathbf{n}\right),
\end{aligned}
$$

for all $\mathbf{v} \in H_{f}, q \in Q, \psi \in H_{p}$.

In order to reduce the computational cost, we might consider the modified Newton method: find $\mathbf{u}_{f}^{n} \in H_{f}, p_{f}^{n} \in Q, \varphi^{n} \in H_{p}$ such that

$$
\begin{aligned}
& a_{f}\left(\mathbf{u}_{f}^{n}, \mathbf{v}\right)+c_{f}\left(\mathbf{u}_{f}^{n} ; \mathbf{u}_{f}^{0}, \mathbf{v}\right)+c_{f}\left(\mathbf{u}_{f}^{0} ; \mathbf{u}_{f}^{n}, \mathbf{v}\right)+b_{f}\left(\mathbf{v}, p_{f}^{n}\right)+\int_{\Gamma} g \varphi^{n}(\mathbf{v} \cdot \mathbf{n}) \\
& \quad+\int_{\Gamma} \sum_{j=1}^{d-1} \frac{\nu}{\varepsilon}\left(\mathbf{u}_{f}^{n} \cdot \boldsymbol{\tau}_{j}\right)\left(\mathbf{v} \cdot \boldsymbol{\tau}_{j}\right)=c_{f}\left(\mathbf{u}_{f}^{n-1} ; \mathbf{u}_{f}^{0}, \mathbf{v}\right) \\
& \quad+c_{f}\left(\mathbf{u}_{f}^{0}-\mathbf{u}_{f}^{n-1} ; \mathbf{u}_{f}^{n-1}, \mathbf{v}\right)+\int_{\Omega_{f}} \mathbf{f} \cdot \mathbf{v} \\
& b_{f}\left(\mathbf{u}_{f}^{n}, q\right)=0 \\
& a_{p}\left(\varphi^{n}, \psi\right)=\int_{\Gamma} \psi\left(\mathbf{u}_{f}^{n} \cdot \mathbf{n}\right)
\end{aligned}
$$

for all $\mathbf{v} \in H_{f}, q \in Q, \psi \in H_{p}$.

Like for fixed-point iterations, we have to solve a linearized coupled problem at each iteration of the Newton algorithms.

We would like to rewrite the Newton methods (78)-(80) and (81)-(83) as iterative schemes for the interface equation (57). Let us consider the exact Newton method. First of all, notice that it can be expressed in the equivalent form:

find $\mathbf{u}_{f}^{n} \in V_{f}, \varphi^{n} \in H_{p}$ such that

$$
\begin{aligned}
& a_{f}\left(\mathbf{u}_{f}^{n}, \mathbf{v}\right)+c_{f}\left(\mathbf{u}_{f}^{n} ; \mathbf{u}_{f}^{n-1}, \mathbf{v}\right)+c_{f}\left(\mathbf{u}_{f}^{n-1} ; \mathbf{u}_{f}^{n}, \mathbf{v}\right)+\int_{\Gamma} g \varphi^{n}(\mathbf{v} \cdot \mathbf{n}) \\
& \quad+\int_{\Gamma} \sum_{j=1}^{d-1} \frac{\nu}{\varepsilon}\left(\mathbf{u}_{f}^{n} \cdot \boldsymbol{\tau}_{j}\right)\left(\mathbf{v} \cdot \boldsymbol{\tau}_{j}\right)=c_{f}\left(\mathbf{u}_{f}^{n-1} ; \mathbf{u}_{f}^{n-1}, \mathbf{v}\right)+\int_{\Omega_{f}} \mathbf{f} \cdot \mathbf{v}, \\
& a_{p}\left(\varphi^{n}, \psi\right)=\int_{\Gamma} \psi\left(\mathbf{u}_{f}^{n} \cdot \mathbf{n}\right),
\end{aligned}
$$


for all $\mathbf{v} \in V_{f}, \psi \in H_{p}$.

Furthermore, (84) can be equivalently restated as: find $\mathbf{u}_{f}^{n} \in V_{f}$ such that

$$
\begin{aligned}
& a_{f}\left(\mathbf{u}_{f}^{n}-\mathbf{u}_{f}^{n-1}, \mathbf{v}\right)+c_{f}\left(\mathbf{u}_{f}^{n}-\mathbf{u}_{f}^{n-1} ; \mathbf{u}_{f}^{n-1}, \mathbf{v}\right)+c_{f}\left(\mathbf{u}_{f}^{n-1} ; \mathbf{u}_{f}^{n}-\mathbf{u}_{f}^{n-1}, \mathbf{v}\right) \\
& +\int_{\Gamma} g\left(\varphi^{n}-\varphi^{n-1}\right)(\mathbf{v} \cdot \mathbf{n})+\int_{\Gamma} \sum_{j=1}^{d-1} \frac{\nu}{\varepsilon}\left(\left(\mathbf{u}_{f}^{n}-\mathbf{u}_{f}^{n-1}\right) \cdot \boldsymbol{\tau}_{j}\right)\left(\mathbf{v} \cdot \boldsymbol{\tau}_{j}\right)= \\
& -a_{f}\left(\mathbf{u}_{f}^{n-1}, \mathbf{v}\right)-c_{f}\left(\mathbf{u}_{f}^{n-1} ; \mathbf{u}_{f}^{n-1}, \mathbf{v}\right)-\int_{\Gamma} g \varphi^{n-1}(\mathbf{v} \cdot \mathbf{n}) \\
& -\int_{\Gamma} \sum_{j=1}^{d-1} \frac{\nu}{\varepsilon}\left(\mathbf{u}_{f}^{n-1} \cdot \boldsymbol{\tau}_{j}\right)\left(\mathbf{v} \cdot \boldsymbol{\tau}_{j}\right)+\int_{\Omega_{f}} \mathbf{f} \cdot \mathbf{v}, \quad \forall \mathbf{v} \in V_{f}
\end{aligned}
$$

Let us now indicate by $P(\bar{u})$ the operator associated to $(57): P(\bar{u}): W \rightarrow W^{\prime}$, $P(\bar{u})=(\tilde{\mathcal{A}} \bar{u}) \bar{u}-\tilde{\ell}, \bar{u}=(\lambda, \mathbf{u}) \in W, \tilde{\mathcal{A}}$ and $\tilde{\ell}$ being defined in Sect. 3. More precisely,

$$
\begin{aligned}
\langle P(\bar{u}), \bar{w}\rangle= & a_{f}\left(\boldsymbol{R}_{f}^{1} \lambda, \boldsymbol{R}_{f}^{1} \mu\right)+a_{f}(\mathbf{u}, \mathbf{w})+c_{f}\left(\mathbf{u}+\boldsymbol{R}_{f}^{1} \lambda ; \mathbf{u}+\boldsymbol{R}_{f}^{1} \lambda, \mathbf{w}+\boldsymbol{R}_{f}^{1} \mu\right) \\
& +\int_{\Gamma} \sum_{j=1}^{d-1} \frac{\nu}{\varepsilon}\left(\boldsymbol{R}_{f}^{1} \lambda \cdot \boldsymbol{\tau}_{j}\right)\left(\boldsymbol{R}_{f}^{1} \mu \cdot \boldsymbol{\tau}_{j}\right)+\int_{\Gamma} \sum_{j=1}^{d-1} \frac{\nu}{\varepsilon}\left(\mathbf{u} \cdot \boldsymbol{\tau}_{j}\right)\left(\mathbf{w} \cdot \boldsymbol{\tau}_{j}\right) \\
& +\int_{\Gamma} g\left(R_{p} \lambda\right) \mu-\int_{\Omega_{f}} \mathbf{f} \cdot\left(\mathbf{w}+\boldsymbol{R}_{f}^{1} \mu\right), \quad \forall \bar{w}=(\mu, \mathbf{w}) \in W .
\end{aligned}
$$

The Gateaux derivative of the operator $P$ in $\bar{u}$ reads, for all $\bar{v}=(\eta, \mathbf{v}), \bar{w}=$ $(\mu, \mathbf{w}) \in W$,

$$
\begin{aligned}
\left\langle\left(P^{\prime}(\bar{u})\right)(\bar{v}), \bar{w}\right\rangle= & a_{f}\left(\boldsymbol{R}_{f}^{1} \eta, \boldsymbol{R}_{f}^{1} \mu\right)+a_{f}(\mathbf{v}, \mathbf{w}) \\
& +c_{f}\left(\mathbf{v}+\boldsymbol{R}_{f}^{1} \eta ; \mathbf{u}+\boldsymbol{R}_{f}^{1} \lambda, \mathbf{w}+\boldsymbol{R}_{f}^{1} \mu\right) \\
& +c_{f}\left(\mathbf{u}+\boldsymbol{R}_{f}^{1} \lambda ; \mathbf{v}+\boldsymbol{R}_{f}^{1} \eta, \mathbf{w}+\boldsymbol{R}_{f}^{1} \mu\right) \\
& +\int_{\Gamma} \sum_{j=1}^{d-1} \frac{\nu}{\varepsilon}\left(\boldsymbol{R}_{f}^{1} \eta \cdot \boldsymbol{\tau}_{j}\right)\left(\boldsymbol{R}_{f}^{1} \mu \cdot \boldsymbol{\tau}_{j}\right) \\
& +\int_{\Gamma} \sum_{j=1}^{d-1} \frac{\nu}{\varepsilon}\left(\mathbf{v} \cdot \boldsymbol{\tau}_{j}\right)\left(\mathbf{w} \cdot \boldsymbol{\tau}_{j}\right)+\int_{\Gamma} g\left(R_{p} \eta\right) \mu .
\end{aligned}
$$

Notice also that, in view of the definition of $\boldsymbol{R}_{f}^{1}$, we have

$$
\begin{aligned}
\langle P(\bar{u}), \bar{w}\rangle= & a_{f}\left(\mathbf{u}+\boldsymbol{R}_{f}^{1} \lambda, \mathbf{w}+\boldsymbol{R}_{f}^{1} \mu\right)+c_{f}\left(\mathbf{u}+\boldsymbol{R}_{f}^{1} \lambda ; \mathbf{u}+\boldsymbol{R}_{f}^{1} \lambda, \mathbf{w}+\boldsymbol{R}_{f}^{1} \mu\right) \\
& +\int_{\Gamma} \sum_{j=1}^{d-1} \frac{\nu}{\varepsilon}\left(\left(\mathbf{u}+\boldsymbol{R}_{f}^{1} \lambda\right) \cdot \boldsymbol{\tau}_{j}\right)\left(\left(\mathbf{w}+\boldsymbol{R}_{f}^{1} \mu\right) \cdot \boldsymbol{\tau}_{j}\right) \\
& +\int_{\Gamma} g\left(R_{p} \lambda\right) \mu-\int_{\Omega_{f}} \mathbf{f} \cdot\left(\mathbf{w}+\boldsymbol{R}_{f}^{1} \mu\right),
\end{aligned}
$$


and

$$
\begin{aligned}
\left\langle\left(P^{\prime}(\bar{u})\right)(\bar{v}), \bar{w}\right\rangle= & a_{f}\left(\mathbf{v}+\boldsymbol{R}_{f}^{1} \eta, \mathbf{w}+\boldsymbol{R}_{f}^{1} \mu\right)+c_{f}\left(\mathbf{v}+\boldsymbol{R}_{f}^{1} \eta ; \mathbf{u}+\boldsymbol{R}_{f}^{1} \lambda, \mathbf{w}+\boldsymbol{R}_{f}^{1} \mu\right) \\
& +c_{f}\left(\mathbf{u}+\boldsymbol{R}_{f}^{1} \lambda ; \mathbf{v}+\boldsymbol{R}_{f}^{1} \eta, \mathbf{w}+\boldsymbol{R}_{f}^{1} \mu\right) \\
& +\int_{\Gamma} \sum_{j=1}^{d-1} \frac{\nu}{\varepsilon}\left(\left(\mathbf{v}+\boldsymbol{R}_{f}^{1} \eta\right) \cdot \boldsymbol{\tau}_{j}\right)\left(\left(\mathbf{w}+\boldsymbol{R}_{f}^{1} \mu\right) \cdot \boldsymbol{\tau}_{j}\right)+\int_{\Gamma} g\left(R_{p} \eta\right) \mu .
\end{aligned}
$$

Following the same argument used in Sect. 4.1, we can write in (86) $\mathbf{u}_{f}^{k}=$ $\mathbf{u}^{k}+\boldsymbol{R}_{f}^{1} \lambda^{k}$ with $\mathbf{u}^{k} \in V_{f}^{0}$ and $\lambda^{k}=\mathbf{u}_{f}^{k} \cdot \mathbf{n}$ on $\Gamma$, and we can set $\varphi^{k}=R_{p} \lambda^{k}$ $(k=n-1, n)$. Moreover, using again the fact that $V_{f}=V_{f}^{0}+\left\{\boldsymbol{R}_{f}^{1} \mu: \mu \in \Lambda_{0}\right\}$, we can write $\mathbf{v}=\mathbf{w}+\boldsymbol{R}_{f}^{1} \mu$ for $\mathbf{w} \in V_{f}^{0}$ and $\mu=\mathbf{v} \cdot \mathbf{n} \in \Lambda_{0}$ on $\Gamma$.

Substituting into (86), we can easily see that it corresponds to the following Newton method to solve (57): given $\bar{u}^{0}=\left(\lambda^{0}, \mathbf{u}^{0}\right) \in W$, for $n \geq 1$, find $\bar{u}^{n}=$ $\left(\lambda^{n}, \mathbf{u}^{n}\right) \in W$ such that

$$
\left\langle\left(P^{\prime}\left(\bar{u}^{n-1}\right)\right)\left(\bar{u}^{n}-\bar{u}^{n-1}\right), \bar{w}\right\rangle=-\left\langle P\left(\bar{u}^{n-1}\right), \bar{w}\right\rangle \quad \forall \bar{w}=(\mu, \mathbf{w}) \in W .
$$

Proceeding in an analogous way, one can show that algorithm (81)-(83) corresponds to the modified Newton method to solve (57): given $\bar{u}^{0}=\left(\lambda_{0}, \mathbf{u}^{0}\right) \in W$, for $n \geq 1$, find $\bar{u}^{n}=\left(\lambda^{n}, \mathbf{u}^{n}\right) \in W$ such that

$$
\left\langle\left(P^{\prime}\left(\bar{u}^{0}\right)\right)\left(\bar{u}^{n}-\bar{u}^{n-1}\right), \bar{w}\right\rangle=-\left\langle P\left(\bar{u}^{n-1}\right), \bar{w}\right\rangle \quad \forall \bar{w}=(\mu, \mathbf{w}) \in W .
$$

Concerning the convergence of the Newton methods we can prove the following result.

Proposition 4.2 Let $\mathbf{f} \in L^{2}\left(\Omega_{f}\right)$ and let

$$
\tilde{C}_{1}=\frac{32 C_{\mathcal{N}} C_{\Omega_{f}}\|\mathbf{f}\|_{0}}{\left(C_{\kappa} \nu\right)^{2}}, \quad \tilde{C}_{2}=\frac{2 \sqrt{2} C_{\Omega_{f}}\|\mathbf{f}\|_{0}}{C_{\kappa} \nu} .
$$

If

$$
\tilde{C}_{1} \leq \frac{1}{2}
$$

then, there exists a unique solution $\bar{u}=\left(\lambda, \mathcal{R}_{0}^{1}(\lambda)\right) \in \bar{B}_{r_{0}}$ of (57), with

$$
\bar{B}_{r_{0}}=\left\{\bar{w}=(\eta, \mathbf{w}) \in W:\|\bar{w}\|_{W} \leq r_{0}\right\}
$$

and

$$
r_{0}=\frac{1-\sqrt{1-2 \tilde{C}_{1}}}{\tilde{C}_{1}} \tilde{C}_{2} .
$$

Moreover, the sequence $\bar{u}^{n}=\left(\lambda^{n}, \mathbf{u}^{n}\right), n \geq 1$, obtained by the Newton algorithms (87) or (88), taking $\bar{u}^{0}=(0, \mathbf{0}) \in W$, converges to this solution.

The following error estimate hold for the Newton method:

$$
\left\|\bar{u}-\bar{u}^{n}\right\|_{W} \leq \frac{1}{2^{n}}\left(2 \tilde{C}_{1}\right)^{2^{n}} \frac{\tilde{C}_{2}}{\tilde{C}_{1}}, \quad n \geq 0,
$$

while for the modified Newton method we have (if $\tilde{C}_{1}<1 / 2$ ):

$$
\left\|\bar{u}-\bar{u}^{n}\right\|_{W} \leq \frac{\tilde{C}_{2}}{\tilde{C}_{1}}\left(1-\sqrt{1-2 \tilde{C}_{1}}\right)^{n+1}, \quad n \geq 0 .
$$


Proof. The proof is a corollary of Theorem 6.3. Consider $\bar{u}^{0}=\left(\lambda^{0}, \mathbf{u}^{0}\right)=$ $(0, \mathbf{0}) \in W$. Then, for all $\bar{w}=(\eta, \mathbf{w}) \in W$, we have:

$$
\begin{aligned}
\left\langle\left(P^{\prime}\left(\bar{u}^{0}\right)\right)(\bar{w}), \bar{w}\right\rangle= & a_{f}\left(\boldsymbol{R}_{f}^{1} \eta, \boldsymbol{R}_{f}^{1} \eta\right)+a_{f}(\mathbf{w}, \mathbf{w})+\int_{\Gamma} \sum_{j=1}^{d-1} \frac{\nu}{\varepsilon}\left(\boldsymbol{R}_{f}^{1} \eta \cdot \boldsymbol{\tau}_{j}\right)\left(\boldsymbol{R}_{f}^{1} \eta \cdot \boldsymbol{\tau}_{j}\right) \\
& +\int_{\Gamma} \sum_{j=1}^{d-1} \frac{\nu}{\varepsilon}\left(\mathbf{w} \cdot \boldsymbol{\tau}_{j}\right)\left(\mathbf{w} \cdot \boldsymbol{\tau}_{j}\right)+\int_{\Gamma} g\left(R_{p} \eta\right) \eta \\
\geq & \frac{C_{\kappa} \nu}{2}\left(\left|\boldsymbol{R}_{f}^{1} \eta\right|_{1}^{2}+|\mathbf{w}|_{1}^{2}\right)=\frac{C_{\kappa} \nu}{2}\|\bar{w}\|_{W}^{2}
\end{aligned}
$$

Consequently, $\left[P^{\prime}\left(\bar{u}_{0}\right)\right]^{-1}$ exists and

$$
\left\|\left[P^{\prime}\left(\bar{u}^{0}\right)\right]^{-1}\right\|_{\mathcal{L}\left(W^{\prime}, W\right)} \leq \frac{2}{C_{\kappa} \nu} .
$$

Moreover,

$$
\left\langle P\left(\bar{u}^{0}\right), \bar{w}\right\rangle=-\int_{\Omega_{f}} \mathbf{f} \cdot\left(\mathbf{w}+\boldsymbol{R}_{f}^{1} \eta\right) \leq \sqrt{2} C_{\Omega_{f}}\|\mathbf{f}\|_{0}\|\bar{w}\|_{W},
$$

and therefore,

$$
\left\|P\left(\bar{u}^{0}\right)\right\|_{W^{\prime}} \leq \sqrt{2} C_{\Omega_{f}}\|\mathbf{f}\|_{0} .
$$

The second derivative of the operator $P$ reads:

$$
\begin{aligned}
\left\langle\left(\left(P^{\prime \prime}(\bar{u})\right)(\bar{v})\right)(\bar{w}), \bar{\zeta}\right\rangle= & c_{f}\left(\mathbf{w}+\boldsymbol{R}_{f}^{1} \eta ; \mathbf{v}+\boldsymbol{R}_{f}^{1} \mu, \mathbf{z}+\boldsymbol{R}_{f}^{1} \xi\right) \\
& +c_{f}\left(\mathbf{v}+\boldsymbol{R}_{f}^{1} \mu ; \mathbf{w}+\boldsymbol{R}_{f}^{1} \eta, \mathbf{z}+\boldsymbol{R}_{f}^{1} \xi\right)
\end{aligned}
$$

for $\bar{u}=(\lambda, \mathbf{u}), \bar{v}=(\mu, \mathbf{v}), \bar{w}=(\eta, \mathbf{w}), \bar{\zeta}=(\xi, \mathbf{z}) \in W$. Thus,

$$
\begin{aligned}
\left\langle\left(\left(P^{\prime \prime}(\bar{u})\right)(\bar{v})\right)(\bar{w}), \bar{\zeta}\right\rangle & \leq 2 C_{\mathcal{N}}\left|\mathbf{w}+\boldsymbol{R}_{f}^{1} \eta\right|_{1}\left|\mathbf{v}+\boldsymbol{R}_{f}^{1} \mu\right|_{1}\left|\mathbf{z}+\boldsymbol{R}_{f}^{1} \xi\right|_{1} \\
& \leq 4 \sqrt{2} C_{\mathcal{N}}|\bar{v}|_{W}|\bar{w}|_{W}|\bar{\zeta}|_{W},
\end{aligned}
$$

so that

$$
\left\|P^{\prime \prime}(\bar{u})\right\|_{\mathcal{L}\left(W, \mathcal{L}\left(W, W^{\prime}\right)\right)} \leq 4 \sqrt{2} C_{\mathcal{N}} .
$$

Consequently, in our case, inequality (106) corresponds to (90).

Moreover, since the operator $P$ is defined and has continuous second derivative on $W$, we can select a radius $r$ satisfying (107)-(110) with $r_{0}$ in (92) and

$$
r_{1}=\frac{1+\sqrt{1-2 \tilde{C}_{1}}}{\tilde{C}_{1}} \tilde{C}_{2}
$$

Finally, the error estimates (93) and (94) are directly obtained from (111) and (112), respectively.

Remark 4.1 With the help of a little algebra we can see that $\tilde{C}_{1}$ and $\tilde{C}_{2}$ are related to the constants $C_{1}$ and $C_{2}$ in (60) as: $C_{1}=2 \sqrt{2} \tilde{C}_{2} / \tilde{C}_{1}$ and $C_{2}=$ $2 \sqrt{2} \tilde{C}_{2}^{2} / \tilde{C}_{1}$. Thus, condition (58) can be reformulated as $\tilde{C}_{1} \leq(3+2 \sqrt{2}) / 8$. If we compare it with (90), we can see that the condition required for the convergence of the Newton method is more restrictive than condition (58) 
Finally, notice that $r_{m}$ becomes

$$
r_{m}=\frac{1-\sqrt{1-\sqrt{2} \tilde{C}_{1}}}{\tilde{C}_{1}} \sqrt{2} \tilde{C}_{2}
$$

Thus, $r_{m}$ has a form similar to $r_{0}$ in (92) and $r_{0} \geq r_{m}$. Notice however that in the definition of $\bar{B}_{r_{m}}$ (see (62)) we control only $\left|\boldsymbol{R}_{f}^{1} \lambda\right|_{1}$, while in $\bar{B}_{r_{0}}$ in (91) we take the whole norm $\|\bar{u}\|_{W}$. We can conclude that the well-posedness results of Lemma 3.1 and Proposition 4.2 are consistent.

\subsection{Preconditioned Richardson method}

We consider the following iterative method to solve (16)-(18): given $\mathbf{u}_{f}^{0} \in H_{f}$, $\varphi^{0} \in H_{p}$, for $n \geq 1$, find $\mathbf{u}_{f}^{n} \in H_{f}, q_{f}^{n} \in Q, \varphi^{n} \in H_{p}$ such that

$$
\begin{aligned}
& a_{f}\left(\mathbf{u}_{f}^{n}-\mathbf{u}_{f}^{n-1}, \mathbf{v}\right)+b_{f}\left(\mathbf{v}, p_{f}^{n}-p_{f}^{n-1}\right)+\int_{\Gamma} \sum_{j=1}^{d-1} \frac{\nu}{\varepsilon}\left(\left(\mathbf{u}_{f}^{n}-\mathbf{u}_{f}^{n-1}\right) \cdot \boldsymbol{\tau}_{j}\right)\left(\mathbf{v} \cdot \boldsymbol{\tau}_{j}\right) \\
& =\theta\left[\int_{\Omega_{f}} \mathbf{f} \cdot \mathbf{v}-a_{f}\left(\mathbf{u}_{f}^{n-1}, \mathbf{v}\right)-c_{f}\left(\mathbf{u}_{f}^{n-1} ; \mathbf{u}_{f}^{n-1}, \mathbf{v}\right)-b_{f}\left(\mathbf{v}, p_{f}^{n-1}\right)\right. \\
& \left.\quad-\int_{\Gamma} \sum_{j=1}^{d-1} \frac{\nu}{\varepsilon}\left(\mathbf{u}_{f}^{n-1} \cdot \boldsymbol{\tau}_{j}\right)\left(\mathbf{v} \cdot \boldsymbol{\tau}_{j}\right)-\int_{\Gamma} g \varphi^{n-1}(\mathbf{v} \cdot \mathbf{n})\right] \\
& b_{f}\left(\mathbf{u}_{f}^{n}-\mathbf{u}_{f}^{n-1}, q\right)=0, \\
& a_{p}\left(\varphi^{n}, \psi\right)=\int_{\Gamma} \psi\left(\mathbf{u}_{f}^{n} \cdot \mathbf{n}\right),
\end{aligned}
$$

for all $\mathbf{v} \in H_{f}, q \in Q, \psi \in H_{p} . \theta>0$ is a suitably chosen relaxation parameter. Unlike the fixed-point and the Newton methods, this algorithm requires to solve at each iteration two decoupled linear equations at each iteration: one in the fluid domain and one in the porous media subdomain.

Proceeding as in Sects. 4.1 and 4.2, we can interpret (97)-(98) as an iterative method for the interface problem (57) and we can prove its convergence for $\theta$ chosen in a suitable interval $\left(0, \theta_{\max }\right)$ with $\theta_{\max }$ depending on $\nu, g$ and $\|\mathbf{f}\|_{0}$. For details we refer the reader to [2].

\section{Some numerical experiments}

We consider the computational domain $\Omega=(0,1) \times(0,2)$ with $\Omega_{f}=(0,1) \times(1,2)$ and $\Omega_{p}=(0,1) \times(0,1)$, and uniform regular triangulations characterized by a parameter $h$. We use Taylor-Hood elements for the Navier-Stokes equations and quadratic Lagrangian elements for the Darcy equation (6).

In a first test, we set the boundary conditions in such a way that the analytical solution for the coupled problem is $\mathbf{u}_{f}=\left(e^{x+y}+y,-e^{x+y}-x\right), p_{f}=$ $\cos (\pi x) \cos (\pi y)+x, \varphi=e^{x+y}-\cos (\pi x)+x y$. In order to check the behavior of the iterative methods that we have studied with respect to the grid parameter $h$, to start with we set the physical parameters $(\nu, \mathrm{K}, \varepsilon, g)$ all equal to 1. 
The algorithms are stopped as soon as $\left\|\mathbf{x}^{n}-\mathbf{x}^{n-1}\right\|_{2} /\left\|\mathbf{x}^{n}\right\|_{2} \leq 10^{-10}$, where $\|\cdot\|_{2}$ is the Euclidean norm and $\mathbf{x}^{n}$ is the vector of the nodal values of $\left(\mathbf{u}_{f}^{n}, p_{f}^{n}, \varphi^{n}\right)$. Our initial guess is $\mathbf{u}_{f}^{0}=\mathbf{0}$.

The number of iterations obtained using the fixed-point algorithm (74)-(76), the Newton method (78)-(80), and the Richardson method (97)-(99) are displayed in table 1. All methods converge in a number of iterations which does not depend on $h$.

Table 1: Number of iterations for the iterative methods with respect to $h$.

\begin{tabular}{cccc}
\hline$h$ & Fixed-point & Newton & Richardson $(\theta=0.5)$ \\
\hline $1 / 7$ & 11 & 5 & 72 \\
$1 / 14$ & 11 & 5 & 72 \\
$1 / 28$ & 11 & 5 & 72 \\
\hline
\end{tabular}

In Fig. 2 (left), we show the convergence history of the three methods in the case $h=1 / 14$, while in Fig. 2 (right) we plot the errors with respect to $h$ between the exact solution and the solution obtained by the Newton method. We can see that the theoretical estimates (72) and (73) are fulfilled.
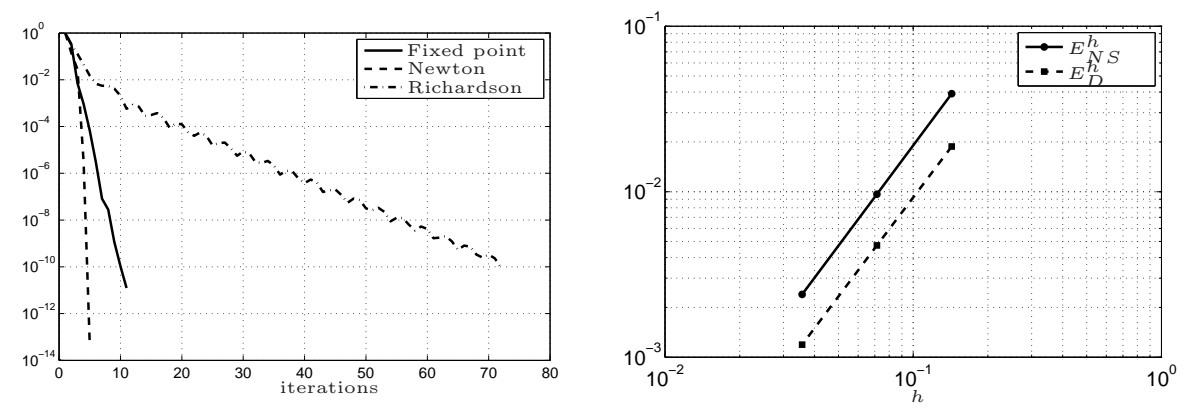

Figure 2: Convergence history for the three methods corresponding to $h=1 / 14$ (left) and errors with respect to the exact solutions (right).

A second test is carried out in order to assess the influence of the physical parameters on the convergence rate of the algorithms. In this case, we consider the same computational domain, however the analytical solution now is $\mathbf{u}_{f}=$ $\left((y-1)^{2}+(y-1)+\varepsilon, x(x-1)\right), p_{f}=2 \nu(x+y-1)$, and $\varphi=\mathrm{K}^{-1}(x(1-x)(y-1)+$ $\left.(y-1)^{3} / 3\right)+2 \nu x$. We choose several values for the physical parameters $\nu$ and $\mathrm{K}$ as indicated in table 2 , and we set $\varepsilon=\sqrt{\mathrm{K}} / 10$. The numerical results in table 2 show that the smaller the parameters the higher the number of iterations. This is particularly evident for the Richardson method where the relaxation parameter $\theta$ must be chosen very small.

In Fig. 3 we show the convergence history of the different methods for $h=1 / 14$ and $\nu=10^{-1}, \mathrm{~K}=10^{-1}$ (on the left), and $\nu=10^{-2}, \mathrm{~K}=10^{-1}$ (on the right).

Concerning the computational cost, we remark that the fixed-point algorithm requires at each iteration to assemble the matrix corresponding to the linearized form $c_{f}\left(\mathbf{u}_{f}^{n} ; \mathbf{u}_{f}^{n-1}, \mathbf{v}\right)$. 
Table 2: Convergence behavior of the iterative methods with respect to the parameters $\nu$ and $\mathrm{K}$.

\begin{tabular}{ccccc}
\multicolumn{4}{c}{ Number of iterations for the fixed-point method } \\
\hline$\nu$ & $\mathrm{K}$ & $h=1 / 7$ & $h=1 / 14$ & $h=1 / 28$ \\
\hline 1 & 1 & 7 & 7 & 7 \\
1 & $10^{-4}$ & 5 & 5 & 5 \\
$10^{-1}$ & $10^{-1}$ & 10 & 10 & 10 \\
$10^{-2}$ & $10^{-1}$ & 17 & 17 & 17 \\
$10^{-2}$ & $10^{-3}$ & 14 & 14 & 14 \\
\hline
\end{tabular}

Number of iterations for the Newton method

\begin{tabular}{ccccc}
\hline$\nu$ & $\mathrm{K}$ & $h=1 / 7$ & $h=1 / 14$ & $h=1 / 28$ \\
\hline 1 & 1 & 5 & 5 & 5 \\
1 & $10^{-4}$ & 4 & 4 & 4 \\
$10^{-1}$ & $10^{-1}$ & 5 & 5 & 5 \\
$10^{-2}$ & $10^{-1}$ & 6 & 6 & 6 \\
$10^{-2}$ & $10^{-3}$ & 5 & 5 & 5 \\
\hline
\end{tabular}

\begin{tabular}{|c|c|c|c|c|c|}
\hline \multicolumn{6}{|c|}{ Number of iterations for the Richardson method } \\
\hline 1 & 1 & 0.5 & 33 & 33 & 33 \\
\hline 1 & $10^{-4}$ & 0.01 & * & $*$ & * \\
\hline $10^{-1}$ & $10^{-1}$ & 0.5 & 44 & 44 & 44 \\
\hline $10^{-2}$ & $10^{-1}$ & 0.01 & $*$ & $*$ & * \\
\hline $10^{-2}$ & $10^{-3}$ & 0.001 & * & * & $*$ \\
\hline
\end{tabular}

On the other hand, at each iteration $n \geq 1$ of the Newton method we have to assemble two matrices associated to the linearized forms $c_{f}\left(\mathbf{u}_{f}^{n} ; \mathbf{u}_{f}^{n-1}, \mathbf{v}\right)$ and $c_{f}\left(\mathbf{u}_{f}^{n-1} ; \mathbf{u}_{f}^{n-1}, \mathbf{v}\right)$ besides updating the right-hand side, which requires the multiplication of the matrix of $c_{f}\left(\mathbf{u}_{f}^{n-1} ; \mathbf{w}, \mathbf{v}\right)$ by the vector $\mathbf{u}_{f}^{n-1}$ of the nodal values of the velocity obtained at the previous iteration. For the set-up of the linear system, the Newton method is thus computationally more expensive than the fixed-point one.

In both cases we have to solve a linear system of similar structure and, in particular, we have to deal with both the fluid and the porous-media subproblems at once. Each of the algorithms (74)-(76) and (78)-(80) involves indeed a linearized Navier-Stokes/Darcy problem in $\Omega_{f} \cup \Omega_{p}$.

The set-up of each iteration of the preconditioned Richardson method is much less expensive. Indeed, only a few matrix-vector multiplications are required to update the right-hand side in (97). Then, one has to solve the Stokes equations (97)-(98) in $\Omega_{f}$ and the Darcy problem (99) in $\Omega_{p}$. These two problems can be solved separately in a sequential mode, since only $\mathbf{u}_{f}^{n}$ is needed in (99) while $\varphi^{n}$ does not appear in the formulation of the fluid subproblem.

In terms of computational effort required to perform each iteration, the Richardson scheme is then the cheapest one. In addition, this method is quite attractive for its decoupling property.

In practice, the extra computational effort for the Newton method pays back 

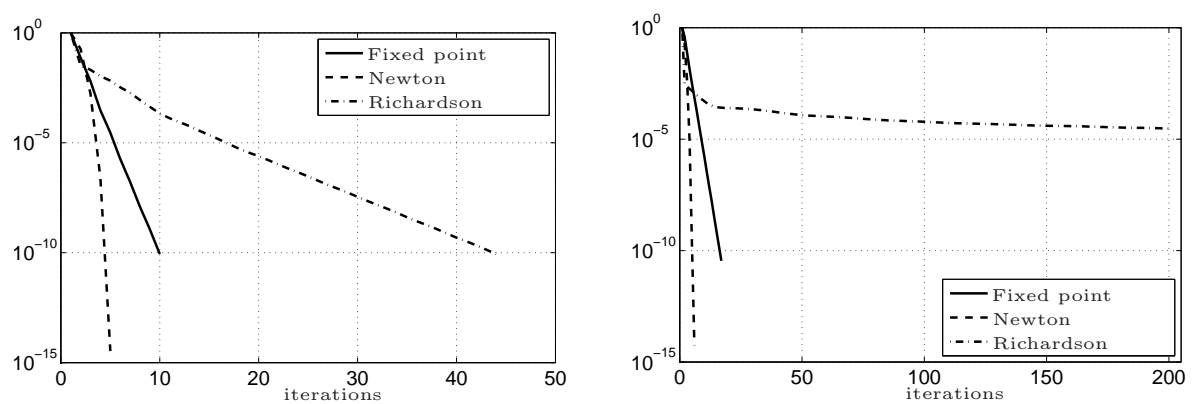

Figure 3: Convergence history for the three methods corresponding to $h=1 / 14$ and $\nu=10^{-1}, \mathrm{~K}=10^{-1}$ (on the left), and $\nu=10^{-2}, \mathrm{~K}=10^{-1}$ (on the right).

with fewer iterations compared to the other algorithms as shown in tables 1 and 2. Moreover, Newton iterations seem to be more robust since their number is substantially independent of both the grid parameter $h$ and of the values of the viscosity $\nu$ and the hydraulic conductivity.

For these reasons, we adopt the Newton method for the following test case.

We consider the computational domain illustrated in Fig. 4 to represent the 2D section of a channel alongside a porous material. In this case, the boundary conditions are chosen in such a way that, if we would disregard the porous media, the Navier-Stokes equations would admit the following Kovasznay solution

$$
\mathbf{u}_{f}=\left(1-e^{\lambda x} \cos (2 \pi y), \frac{\lambda}{2 \pi} e^{\lambda x} \sin (2 \pi y)\right), \quad p_{f}=-\frac{e^{2 \lambda x}}{2},
$$

with $\lambda=0.5 \operatorname{Re}-\sqrt{0.25 \operatorname{Re}^{2}+4 \pi^{2}}, \operatorname{Re}=1 / \nu$ and $\nu=0.025$. Moreover, we impose that the conormal derivative of the piezometric head is null on $\Gamma_{p}^{N}$ and $\varphi=-0.25$ on $\Gamma_{p}^{D}$. The hydraulic conductivity coefficient is $\mathrm{K}=10^{-1}$.

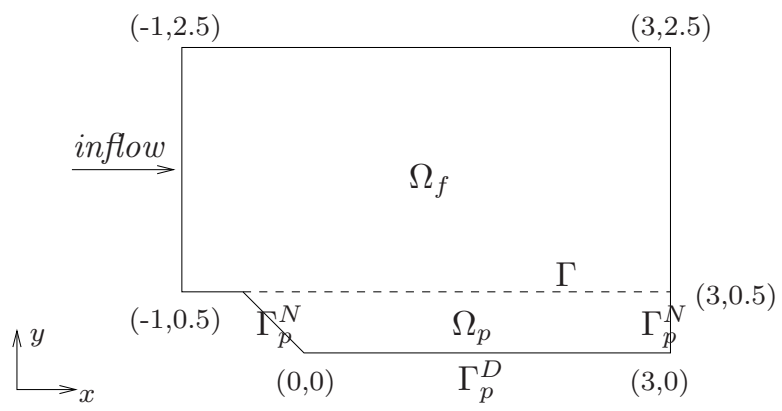

Figure 4: Computational domain for the third test case.

We have solved this problem using two different grids and adopting the Newton method. The convergence results are reported in table 3 , the computed velocity and piezometric head are displayed in Fig. 5. 
Table 3: Newton iterations to solve the problem illustrated in Fig. 4.

\begin{tabular}{cc}
\hline Grid elements & Newton iterations \\
\hline 792 & 7 \\
3168 & 8 \\
\hline
\end{tabular}
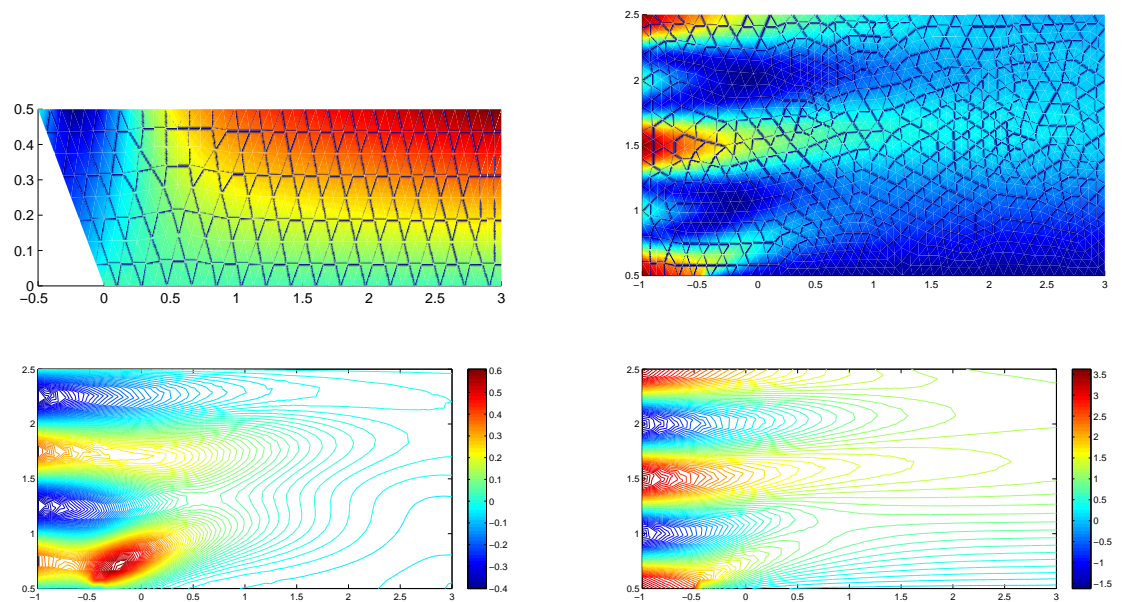

Figure 5: Computed solution: piezometric head $\varphi$ (top left), velocity field $\mathbf{u}_{f}$ (top right), contour lines of the velocity in $x$ - (bottom left) and $y$-direction (bottom right). Notice that the Kovasznay flow is modified due to the presence of the porous media: indeed, the velocity in $y$-direction has negative values in correspondence to the porous media interface.

\section{Appendix}

\subsection{Some existence and uniqueness results}

In this section we recall some existence and uniqueness results for nonlinear saddle-point problems, referring the reader to, e.g., $[5,6,7,9]$ and also [17] for a rigorous study.

Let $\left(X,\|\cdot\|_{X}\right)$ and $\left(Y,\|\cdot\|_{Y}\right)$ be two real Hilbert spaces and consider a bilinear continuous form $\mathrm{b}(\cdot, \cdot): X \times Y \rightarrow \mathbb{R},(v, q) \rightarrow \mathrm{b}(v, q)$, and a trilinear form $\mathrm{a}(\cdot ; \cdot, \cdot): X \times X \times X \rightarrow \mathbb{R},(w, u, v) \rightarrow \mathrm{a}(w ; u, v)$, where, for $w \in X$ the mapping $(u, v) \rightarrow \mathrm{a}(w ; u, v)$ is a bilinear continuous form on $X \times X$.

Then, we consider the following problem: given $l \in X^{\prime}$, find a pair $(u, p) \in X \times Y$ satisfying

$$
\begin{aligned}
\mathrm{a}(u ; u, v)+\mathrm{b}(v, p) & =\langle l, v\rangle & & \forall v \in X \\
\mathrm{~b}(u, q) & =0 & & \forall q \in Y .
\end{aligned}
$$

Introducing the linear operators $A(w) \in \mathcal{L}\left(X ; X^{\prime}\right)$ for $w \in X$, and $B \in \mathcal{L}\left(X ; Y^{\prime}\right)$ :

$$
\begin{aligned}
& \langle A(w) u, v\rangle=\mathrm{a}(w ; u, v) \quad \forall u, v \in X, \\
& \langle B v, q\rangle=\mathrm{b}(v, q) \quad \forall v \in X, \forall q \in Y,
\end{aligned}
$$


problem (100) becomes: find $(u, p) \in X \times Y$ such that

$$
\begin{aligned}
A(u) u+B^{T} p & =l & & \text { in } X^{\prime}, \\
B u & =0 & & \text { in } Y^{\prime} .
\end{aligned}
$$

Taking $V=\operatorname{Ker}(B)$, we associate (100) with the problem

$$
\text { find } u \in V: \quad \mathrm{a}(u ; u, v)=\langle l, v\rangle \quad \forall v \in V,
$$

or, equivalently: find $u \in V$ such that $\Pi A(u) u=\Pi l$ in $V^{\prime}$, where the linear operator $\Pi \in \mathcal{L}\left(X^{\prime} ; V^{\prime}\right)$ is defined by $\langle\Pi l, v\rangle=\langle l, v\rangle, \forall v \in V$.

If $(u, p)$ is a solution of problem (100), then $u$ solves (102). The converse may be proved provided an inf-sup condition holds. Indeed, the following results can be proved.

\section{Theorem 6.1 (Existence and uniqueness) Suppose that:}

1. the bilinear form $\mathrm{a}(w ; \cdot, \cdot)$ is uniformly elliptic in the Hilbert space $V$ with respect to $w$, i.e. there exists a constant $\alpha>0$ such that

$$
\mathrm{a}(w ; v, v) \geq \alpha\|v\|_{X}^{2} \quad \forall v, w \in V
$$

2. the mapping $w \rightarrow \Pi A(w)$ is locally Lipschitz-continuous in $V$, i.e. there exists a continuous and monotonically increasing function $L: \mathbb{R}^{+} \rightarrow \mathbb{R}^{+}$ such that for all $m>0$

$$
\begin{aligned}
& \left|\mathrm{a}\left(w_{1} ; u, v\right)-\mathrm{a}\left(w_{2} ; u, v\right)\right| \leq L(m)\|u\|_{X}\|v\|_{X}\left\|w_{1}-w_{2}\right\|_{X} \\
& \forall u, v \in V, \forall w_{1}, w_{2} \in S_{m} \text { with } S_{m}=\left\{w \in V \mid\|w\|_{X} \leq m\right\} ;
\end{aligned}
$$

3. it holds

$$
\frac{\|\Pi l\|_{V^{\prime}}}{\alpha^{2}} L\left(\frac{\|\Pi l\|_{V^{\prime}}}{\alpha}\right)<1 .
$$

Then (102) has a unique solution $u \in V$.

We consider now problem (100).

Theorem 6.2 Assume that the bilinear form $\mathrm{b}(\cdot, \cdot)$ satisfies the inf-sup condition: $\exists \beta>0$

$$
\inf _{q \in Y} \sup _{v \in X} \frac{\mathrm{b}(v, q)}{\|v\|_{X}\|q\|_{Y}} \geq \beta .
$$

Then for each solution $u$ of (102) there exists a unique $p \in Y$ such that the pair $(u, p)$ is a solution of (100).

\subsection{Convergence of the Newton methods}

Let $X$ and $Y$ be two Banach spaces. We consider the sphere of radius $R>0$ centered in $x^{0} \in X: \Omega=\left\{x \in X:\left\|x-x^{0}\right\|_{X}<R\right\}$, and the closed sphere of radius $0<r<R$ centered in $x^{0}: \Omega_{0}=\left\{x \in X:\left\|x-x^{0}\right\|_{X} \leq r\right\}$. We assume that $\Omega$ contains a zero of an operator $\mathcal{P}: \Omega \subset X \rightarrow Y$, i.e. a point $x^{*} \in \Omega$ such that $\mathcal{P}\left(x^{*}\right)=0$. 
If $\mathcal{P}$ has a continuous derivative in $\Omega$, we can apply the Newton method to compute the zero $x^{*}$ : given an initial approximation $x^{0} \in \Omega$ of $x^{*}$, for $n \geq 0$,

$$
x^{n+1}=x^{n}-\left[\mathcal{P}^{\prime}\left(x^{n}\right)\right]^{-1}\left(\mathcal{P}\left(x^{n}\right)\right),
$$

assuming that $\left[\mathcal{P}^{\prime}\left(x^{n}\right)\right]^{-1}$ exists.

Alternatively, we can use the modified Newton algorithm: given $x^{0} \in \Omega$, for $n \geq 0$,

$$
x^{n+1}=x^{n}-\left[\mathcal{P}^{\prime}\left(x^{0}\right)\right]^{-1}\left(\mathcal{P}\left(x^{n}\right)\right) .
$$

Concerning the convergence of these methods, we have the following theorem (Theorem 6 (1.XVIII) in [21], p. 708).

Theorem 6.3 (Kantorovich Theorem) Let $\mathcal{P}$ be defined on $\Omega \subset X$ with continuous second derivative in $\Omega_{0}$. Moreover assume that

(1) there exists the continuous linear operator $\left[\mathcal{P}^{\prime}\left(x^{0}\right)\right]^{-1}$;

(2) there exists a positive constant $K_{1}>0:\left\|\left[\mathcal{P}^{\prime}\left(x^{0}\right)\right]^{-1}\left(\mathcal{P}\left(x^{0}\right)\right)\right\|_{X} \leq K_{1}$;

(3) there exists a positive constant $K_{2}>0:\left\|\left[\mathcal{P}^{\prime}\left(x^{0}\right)\right]^{-1} \mathcal{P}^{\prime \prime}(x)\right\|_{X} \leq K_{2}$ for all $x \in \Omega_{0}$.

If

$$
K_{3}=K_{1} K_{2} \leq \frac{1}{2},
$$

and the radius $r$ of $\Omega_{0}$ satisfies

$$
r \geq r_{0}=\frac{1-\sqrt{1-2 K_{3}}}{K_{3}} K_{1},
$$

then, there exists a zero $x^{*}$ of $\mathcal{P}$ to which the Newton and the modified Newton methods converge. In this case,

$$
\left\|x^{*}-x^{0}\right\|_{X} \leq r_{0}
$$

Furthermore, if for $K_{3}<1 / 2$

$$
r<r_{1}=\frac{1+\sqrt{1-2 K_{3}}}{K_{3}} K_{1}
$$

or for $K_{3}=1 / 2$

$$
r \leq r_{1}
$$

the solution $x^{*}$ is unique in the sphere $\Omega_{0}$.

The convergence rate of the Newton method is characterized by

$$
\left\|x^{*}-x^{n}\right\|_{X} \leq \frac{1}{2^{n}}\left(2 K_{3}\right)^{2^{n}} \frac{K_{1}}{K_{3}}, \quad n \geq 0
$$

while that of the modified method, for $K_{3}<1 / 2$, by

$$
\left\|x^{*}-x^{n}\right\|_{X} \leq \frac{K_{1}}{K_{3}}\left(1-\sqrt{1-2 K_{3}}\right)^{n+1}, \quad n \geq 0 .
$$


Acknowledgments. The first author acknowledges the financial support from the program SCOPES n. IB7320-110721 and from IACS, EPFL. The other authors acknowledge the support of the FNS Project n. 200020-117587 "Interface operators and solutions algorithms for fluid-structure interaction problems with applications". The second author acknowledges also the Radon Institute for Computational and Applied Mathematics (RICAM), Linz, Austria, for partially supporting this research. Finally, the third author acknowledges also the financial support from the program Cofin MIUR PRIN 2007 n. 200774A7LH_001

"Mathematical and numerical modelling for cardiovascular and fluid dynamics applications".

\section{References}

[1] R.A. Adams. Sobolev Spaces. Academic Press, New York, 1975.

[2] L. Badea, M. Discacciati, and A. Quarteroni. Numerical analysis of the Navier-Stokes/Darcy coupling. Technical report, Ecole Polytechnique Fédérale de Lausanne, IACS-CMCS, 2008.

[3] H. Berninger. Domain Decomposition Methods for Elliptic Problems with Jumping Nonlinearities and Application to the Richards Equation. $\mathrm{PhD}$ thesis, Freie Universität Berlin, 2007.

[4] F. Brezzi and M. Fortin. Mixed and Hybrid Finite Element Method. Springer, New York, 1991.

[5] F. Brezzi, J. Rappaz, and P.A. Raviart. Finite dimensional approximation of nonlinear problems. Part I: Branches of nonlinear solutions. Numer. Math., 36:1-25, 1980.

[6] F. Brezzi, J. Rappaz, and P.A. Raviart. Finite dimensional approximation of nonlinear problems. Part II: Limit points. Numer. Math., 37:1-28, 1981.

[7] F. Brezzi, J. Rappaz, and P.A. Raviart. Finite dimensional approximation of nonlinear problems. Part III: Simple bifurcation points. Numer. Math., $38: 1-30,1981$.

[8] H.C. Brinkman. A calculation of the viscous force exerted by a flowing fluid on a dense swarm of particles. Appl. Sci. Res. A, 1:27-34, 1947.

[9] G. Caloz and J. Rappaz. Numerical Analysis for Nonlinear and Bifurcation Problems. In Handbook of Numerical Analysis, Vol. V, pages 487-637. North-Holland, Amsterdam, 1997.

[10] M. Discacciati. Domain Decomposition Methods for the Coupling of Surface and Groundwater Flows. PhD thesis, Ecole Polytechnique Fédérale de Lausanne, Switzerland, 2004.

[11] M. Discacciati, E. Miglio, and A. Quarteroni. Mathematical and numerical models for coupling surface and groundwater flows. Appl. Numer. Math., 43:57-74, 2002. 
[12] M. Discacciati and A. Quarteroni. Analysis of a domain decomposition method for the coupling of Stokes and Darcy equations. In F. Brezzi, A. Buffa, S. Corsaro, and A. Murli, editors, Numerical Mathematics and Advanced Applications, ENUMATH 2001, pages 3-20. Springer. Milan, 2003.

[13] M. Discacciati and A. Quarteroni. Convergence analysis of a subdomain iterative method for the finite element approximation of the coupling of Stokes and Darcy equations. Comput. Visual. Sci., 6:93-103, 2004.

[14] M. Discacciati and A. Quarteroni. Navier-Stokes/Darcy coupling: modeling, analysis, and numerical approximation. Rev. Mat. Complut., $22(2): 315-426,2009$.

[15] P. Forchheimer. Wasserbewegung durch Boden. Z. Ver. Deutsch. Ing., 45:1782-1788, 1901.

[16] T. Giorgi. Derivation of the Forchheimer law via matched asymptotic expansions. Transport in Porous Media, 29:191-206, 1997.

[17] V. Girault and P.A. Raviart. Finite Element Methods for Navier-Stokes Equations. Theory and Algorithms. Springer, Berlin, 1986.

[18] W. Jäger and A. Mikelić. On the boundary conditions at the contact interface between a porous medium and a free fluid. Ann. Scuola Norm. Sup. Pisa Cl. Sci., 23:403-465, 1996.

[19] W. Jäger and A. Mikelić. On the interface boundary condition of Beavers, Joseph and Saffman. SIAM J. Appl. Math., 60(4):1111-1127, 2000.

[20] W. Jäger, A. Mikelić, and N. Neuss. Asymptotic analysis of the laminar viscous flow over a porous bed. SIAM J. Sci. Comput., 22(6):2006-2028, 2001.

[21] L.V. Kantorovich and G.P. Akilov. Functional Analysis in Normed Spaces. Translated from the Russian by D. E. Brown. Edited by A. P. Robertson. International Series of Monographs in Pure and Applied Mathematics, Vol. 46. The Macmillan Co., New York, 1964.

[22] W.L. Layton, F. Schieweck, and I. Yotov. Coupling fluid flow with porous media flow. SIAM J. Num. Anal., 40:2195-2218, 2003.

[23] J.L. Lions and E. Magenes. Problèmes aux Limites Non Homogènes et Applications, volume 1. Dunod, Paris, 1968.

[24] E. Marušić-Paloka and A. Mikelić. The derivation of a nonlinear filtration law including the inertia effects via homogenization. Nonlin. Anal., 42:97$137,2000$.

[25] A. Quarteroni and A. Valli. Numerical Approximation of Partial Differential Equations. Springer, Berlin, 1994.

[26] A. Quarteroni and A. Valli. Domain Decomposition Methods for Partial Differential Equations. The Clarendon Press, Oxford University Press, New York, 1999. 
[27] K. Yosida. Functional Analysis. Springer, Berlin, 1974.

[28] P. Zunino. Mathematical and Numerical Modeling of Mass Transfer in the Vascular System. PhD thesis, Ecole Polytechnique Fédérale de Lausanne, Switzerland, 2002. 